\title{
Topological Gauge Theories and Group Cohomology
}

\author{
Robbert Dijkgraaf ${ }^{1 \star}$ and Edward Witten ${ }^{2}$ \\ ${ }^{1}$ Institute for Theoretical Physics, University of Utrecht, The Netherlands \\ School of Natural Sciences, Institute for Advanced Study, Olden Lane, Princeton, \\ NJ 08540, USA
}

\begin{abstract}
We show that three dimensional Chern-Simons gauge theories with a compact gauge group $G$ (not necessarily connected or simply connected) can be classified by the integer cohomology group $H^{4}(B G, \mathbf{Z})$. In a similar way, possible Wess-Zumino interactions of such a group $G$ are classified by $H^{3}(G, \mathbf{Z})$. The relation between three dimensional Chern-Simons gauge theory and two dimensional sigma models involves a certain natural map from $H^{4}(B G, \mathbf{Z})$ to $H^{3}(G, \mathbf{Z})$. We generalize this correspondence to topological "spin" theories, which are defined on three manifolds with spin structure, and are related to what might be called $\mathbf{Z}_{2}$ graded chiral algebras (or chiral superalgebras) in two dimensions. Finally we discuss in some detail the formulation of these topological gauge theories for the special case of a finite group, establishing links with two dimensional (holomorphic) orbifold models.
\end{abstract}

\section{Introduction}

Topological gauge field theories in three dimensions are related in an interesting way to two dimensional mathematical physics [1] and are interesting as well for their purely geometrical content. One of the key ingredients in formulating three dimensional topological gauge theories is the Chern-Simons action functional. Thus, let $M$ be an oriented three manifold, $G$ a compact gauge group, $\operatorname{Tr}$ an invariant quadratic form on the Lie algebra of $G$, and $A$ a connection on a $G$ bundle $E$. If $E$ is trivial, the connection $A$ can be regarded as a Lie algebra valued one form, and we can define the Chern-Simons functional by the familiar formula

$$
S(A)=\frac{k}{8 \pi^{2}} \int_{M} \operatorname{Tr}\left(A \wedge d A+\frac{2}{3} A \wedge A \wedge A\right) .
$$

One can then use this functional as the Lagrangian of a quantum field theory. In this paper we use a normalization in which the path integral reads

$$
Z(M)=\int \mathscr{D} A e^{2 \pi i S(A)} .
$$

* Present address: Joseph Henry Laboratories, Princeton University, Princeton, NJ 08544, USA 
The parameter $k$ in (1.1) must be an integer so that the integrand in the path integral is single-valued.

If $G$ is a connected, simply connected compact Lie group, then a $G$ bundle on a three manifold is necessarily trivial, so the above definition of the action is adequate. For more general Lie groups (such as those studied in [2]) non-trivial bundles over $M$ may exist and we will include in the path integral also a summation over all possible bundles $E$. The inclusion of non-trivial bundles actually tells us that we are considering the gauge group $G$ and not a connected, simply connected group whose Lie algebra equals $\operatorname{Lie}(G)$.

If the bundle $E$ is not trivial, the formula (1.1) for the action $S$ does not make sense, since a connection on a non-trivial bundle cannot be represented by a Lie algebra valued one form as in that formula. A more general definition can be obtained as follows. Any three manifold $M$ can be realized as the boundary of a four manifold $B$. If it is possible to choose $B$ so that $E$ extends over $B$ then (upon picking an extension of $A$ over $B$ ) we can define the Chern-Simons functional by the formula

$$
S(A)=\frac{k}{8 \pi^{2}} \int_{B} \operatorname{Tr}(F \wedge F)
$$

A standard argument shows that if $k$ is an integer, $S(A)$ is independent, modulo 1, of the choice of $B$ and of the extensions of $E$ and $A$. Equation (1.3) reduces to (1.1) when (1.1) makes sense, and so does represent a more general definition of the Chern-Simons functional.

Depending on $\pi_{1}(M)$ and $G$, there may exist non-trivial flat connections on $M$. The action $S(A)$ for a flat connection $A$ is in general not zero, but is an interesting invariant of the representation of the fundamental group of $M$ determined by the flat connection $A$. However, (1.3) implies the important fact that

$$
S(A)=0
$$

for a flat connection $A$ which extends as a flat connection over some bounding four manifold $B$. In other words, if $B$ and $E$ and the extension of $A$ can be chosen so that $F=0$ on $B$, then obviously $S(A)=0$.

In general it will be impossible to find a four manifold $B$, with boundary $M$, over which $E$ can be extended, and therefore (1.3) is still not a completely general definition of the topological action. One of our goals in this paper is to give a completely general definition (for an arbitrary compact group $G$, not necessarily connected or simply connected). To understand a bit better the nature of the problem, note that if $A$ and $A^{\prime}$ are two different connections on the same bundle $E$, then (1.3) can always be used to define the difference $S(A)-S\left(A^{\prime}\right)$. In fact, the four manifold $B=M \times I$ has boundary $M \cup(-M)$ (here $(-M)$ is $M$ with opposite orientation). Since $B$ retracts onto $M$, the bundle $E$ has (up to homotopy) a unique extension, which we will also call $E$, over $B$, and it is possible to find a connection $A^{\prime \prime}$ on $B$ that interpolates between $A$ on $M \times\{0\}$ and $A^{\prime}$ on $M \times\{1\}$. So a special case of (1.3) is

$$
S(A)-S\left(A^{\prime}\right)=\frac{k}{8 \pi^{2}} \int_{B} \operatorname{Tr}(F \wedge F)
$$


where $F$ is the curvature of $A^{\prime \prime}$; in fact, by standard arguments the right-hand side of (1.5) depends modulo 1 only on $A$ and $A^{\prime}$ and not on the choice of $A^{\prime \prime}$. Since (1.5) defines the difference $S(A)-S\left(A^{\prime}\right)$ for any two connections $A$ and $A^{\prime}$ on $E$, what remains to be fixed is just an integration constant that depends on $M$ and $E$ but not on the particular choice of a connection $A$. To define a topological quantum field theory, one needs a way to fix these integration constants for all possible three manifolds $M$ and $G$ bundles $E$, in a way compatible with basic physical requirements of unitarity and factorization.

To give an orientation to this problem (and an example which is quite typical of our interests in this paper), consider an example which is of the opposite type from the connected, simply connected groups for which (1.1) serves as an adequate definition of the topological action. Let us consider the case in which $G$ is a finite group. Every principal $G$ bundle has a unique, flat connection, and corresponds to a homomorphism $\lambda: \pi_{1}(M) \rightarrow G$. Since the connections are unique, the integration constants that we previously isolated by using (1.5) are in this case from the beginning all that there is to discuss.

Since we want to be able to consider transition amplitudes between initial and final states (defined on Riemann surfaces), we consider three manifolds $M$ whose boundaries are not necessarily empty. A "topological action" $S$ for the gauge group $G$ would be a rule which to every pair $(M, \lambda)$ (with $M$ being a three manifold and $\lambda$ a homomorphism of $\pi_{1}(M)$ to $G$ ) assigns a value $S(\lambda)$ in $R / \mathbf{Z}$ subject to the following:

(i) Two actions $S$ and $S^{\prime}$ should be considered equivalent if they differ by a functional that only depends on the restriction of $\lambda$ to the boundary of $M$-since in that case the difference between the transition amplitudes $e^{2 \pi i S}$ and $e^{2 \pi i S^{\prime}}$ can be absorbed in a redefinition of the external state wave functions.

(ii) If $M$ has no boundary, and it is possible to find a four manifold $B$ such that $\partial B=M$ (that is, the boundary of $B$ is $M$ ) and such that $\lambda$ extends to a homomorphism $\lambda_{B}: \pi_{1}(B) \rightarrow G$, then we require $S(\lambda)=0$. As we have seen in (1.4), this requirement holds for arbitrary $G$, not just finite groups.

Physically, this requirement amounts to a requirement of factorization. This point may require some discussion. In fact, if $M$ is the connected sum of three manifolds $M_{1}$ and $M_{2}$, one could find a four manifold $B$ of boundary $M \cup\left(-M_{1}\right)$ $\cup\left(-M_{2}\right)$. If one picks $B$ to represent a space-time history of $M$ splitting into $M_{1} \cup M_{2}$, then every $G$ connection $\lambda$ on $M$ extends over $B$, and (1.4) implies

$$
e^{2 \pi i S(M)}=e^{2 \pi i S\left(M_{1}\right)} \cdot e^{2 \pi i S\left(M_{2}\right)},
$$

which is the statement of factorization.

The problem of classifying action functionals $S(\lambda)$ subject to (i), (ii) is a standard problem and the answer is as follows. Such action functionals are in one to one correspondence with elements of the cohomology group $H^{3}(B G, R / \mathbf{Z})$, where $B G$ is the classifying space of the group $G$. These concepts will be explained to some extent in the following sections.

This answer can be reexpressed in the following way. Looking at the long exact sequence in cohomology derived from the exact sequence of groups,

$$
0 \rightarrow \mathbf{Z} \rightarrow R \rightarrow R / \mathbf{Z} \rightarrow 0,
$$


and using the fact that for a finite group, the cohomology with coefficients $R$ vanishes, we find that $H^{k}(B G, R / \mathbf{Z}) \cong H^{k+1}(B G, \mathbf{Z})$. In particular, $H^{3}(B G, R / \mathbf{Z})$ $\cong H^{4}(B G, \mathbf{Z})$. Therefore, we can consider the topological actions for finite groups to be classified by $H^{4}(B G, \mathbf{Z})$.

This way of looking at things is fruitful for the following reason. Let us go back to the case in which $G$ is a connected, simply connected group and the simple definition (1.1) of the topological action is adequate. For a group of this type, the topological actions are classified by the integer $k$ that appears in (1.1) or (1.3). On the other hand, it is also so for connected, simply connected $G$ that $H^{4}(B G, \mathbf{Z}) \cong \mathbf{Z}$. What is more, the generator of $H^{4}(B G, Z)$ corresponds exactly to the characteristic class $\frac{1}{8 \pi^{2}} \operatorname{Tr}(F \wedge F)$ that appears in (1.3). Thus, we can consider the topological actions for connected, simply connected groups to be classified by $H^{4}(B G, \mathbf{Z})$.

Thus, a common answer arises for the two opposite kinds of group - the connected, simply connected ones in which the classification of the components of the space of connections is trivial and the finite groups in which this classification is the whole story (since there is only one connection on any given principal bundle). This strongly suggests that the same result will hold for gauge groups intermediate between these extreme kinds. We will show that this is so - that for an arbitrary compact Lie group $G$ one can construct a topological action corresponding to any element of $H^{4}(B G, \mathbf{Z})$.

One reason that this is natural is that general Lie groups can be built in simple ways from the types considered above. In fact, any Lie group $G$ appears in an exact sequence

$$
1 \rightarrow G_{0} \rightarrow G \rightarrow \Gamma \rightarrow 1,
$$

where $G_{0}$ is the component of the identity and $\Gamma$ is the group of components. If $G$ is compact, then $\Gamma$ is a finite group, one of the two types that we have considered. $G$ also appears in the fundamental exact sequence

$$
1 \rightarrow \pi_{1}(G) \rightarrow \widetilde{G} \rightarrow G \rightarrow 1,
$$

where $\widetilde{G}$ is the simply connected universal cover of $G$. Combining these two exact sequences, any compact $G$ with finite fundamental group is built from a connected, simply connected group and some finite groups - the two extreme cases that we have just considered.

This paper is organized as follows. In Sect. (2) we will briefly review some essentials in the theory of group cohomology that we will need in the subsequent sections. After these preparations we will discuss the construction of topological actions in Sect. (3). In Sect. (4) we will address the relation of these three dimensional topological theories to two dimensional WZW conformal field theories and generalizations of them. Our discussion will be limited here to a derivation of the two dimensional Wess-Zumino actions. These are classified by the classes in $H^{3}(G, \mathbf{Z})$, and we will show that the natural map $H^{4}(B G, \mathbf{Z})$ $\rightarrow H^{3}(G, \mathbf{Z})$ gives us the correspondence. This map is not surjective, and this gives an alternative explanation of why for non-simply-connected groups, chiral algebras only exist at certain particular values of the level $k$ [2]. In fact, the quantization condition on $k$ is completely explained by topological considerations 
in four dimensions. Section (5) contains an extension of the construction of topological gauge theories to the category of spin manifolds. These topological "spin" theories will require a definite choice of spin structure on the manifold in order to be well-defined. They are related in two dimensions to what one might call $\mathbf{Z}_{2}$ graded chiral algebras, or chiral superalgebras. Superconformal field theories are examples of theories with interesting chiral superalgebras, and it seems more natural to think about superconformal field theories as theories with chiral superalgebras than to regard them as theories with chiral algebras in which there just happens to be a primary field of dimension $3 / 2$ with certain interesting properties.

Finally, in Sect. (6) we will return to a theme touched on above - the three dimensional topological theories with finite gauge group. We will show that these theories can be very neatly represented in a form similar to lattice gauge theory. These theories have some claims to being the most simple quantum field theories, being completely finite, and topological in nature. They also provide an elementary but enlightening illustration of the functorial description of quantum field theory along the lines of Segal [6]. We will furthermore establish in some detail the connection between our results and those obtained in the analysis of two dimensional (holomorphic) orbifold models in [7].

\section{Cohomology of Groups}

In order to be more or less self-contained, we will first review some essential ingredients of homology and algebraic topology that we will need in the following sections. We give a very brief review of the singular homology and cohomology theory of topological spaces, in particular of classifying spaces of compact Lie groups, and their relation to characteristic classes. A much more thorough treatment of the material in this section can of course be found in the mathematical literature, for instance in [8-12]; for an introduction to integer homology, aimed at physicists, that stresses the importance of torsion, see [13].

2.1. Singular Cohomology Theory. We will first recall the definition of singular homology with integer coefficients. For any topological space $T$ we can introduce the groups of singular chains $C_{k}(T)$. A singular $k$-chain is essentially a map of a collection of $k$-dimensional simplices into the space $T$. The group operation is simply addition with integer coefficients. One further defines certain subgroups $B_{k}(T)$ and $Z_{k}(T)$ of $C_{k}(T)$. The "boundaries" $B_{k}(T)$ and the "cycles" $Z_{k}(T)$ consist respectively of chains $C$ that satisfy $C=\partial B$ and $\partial C=0$, with $\partial$ the boundary operator. The homology groups are defined as the quotients $H_{k}(T, \mathbf{Z})$ $=Z_{k}(T) / B_{k}(T)$. Completely similarly, one can introduce the space of (integer) cochains

$$
C^{k}(T, \mathbf{Z})=\operatorname{Hom}\left(C_{k}(T), \mathbf{Z}\right),
$$

and with the aid of the coboundary operator $\delta$, coboundaries, cocycles and cohomology groups $H^{k}(T, Z)$. Here the coboundary operator is defined by

$$
\langle\delta \alpha, C\rangle=(-1)^{k}\langle\alpha, \partial C\rangle,
$$


with $\langle\cdot, \cdot\rangle$ the pairing $C_{k}(T) \otimes C^{k}(T) \rightarrow \mathbf{Z}$. The cohomology groups $H^{k}(T, F)$ can be defined with coefficients in any abelian group $F$, by replacing $\mathbf{Z}$ by $F$ in the definition (2.1) of the cochains. In particular, with real coefficients we have $H^{k}(T, R)$ $=H^{k}(T, \mathbf{Z}) \otimes R$. Due to the fundamental theorem of De Rham, these real cocycles can be represented by closed differential forms. We further recall that in the case that $F$ is a divisible group, so in particular for $F=R$, we have another very simple definition of the cohomology groups:

$$
H^{k}(T, F)=\operatorname{Hom}\left(H_{k}(T), F\right),
$$

i.e. $\alpha \in H^{k}(T, F)$ is a homomorphism $Z_{k}(T) \rightarrow F$ that vanishes on boundaries.

An element of finite order of an abelian group is called a torsion element. The homology and cohomology groups of a topological space (with arbitrary coefficients) are abelian groups. The universal coefficient theorem gives an isomorphism (but not a completely natural one) between the torsion of $H_{k-1}(T, \mathbf{Z})$ and that of $H^{k}(T, \mathbf{Z})$. Torsion elements in $H^{k}(T, \mathbf{Z})$ cannot be represented in the usual fashion by differential forms, since torsion classes are elements of the kernel of the map $\varrho: H^{k}(T, \mathbf{Z}) \rightarrow H^{k}(T, R)$. An Abelian group $A$ has a torsion subgroup Tor $A$, but there is no natural map from $A$ to Tor $A$. Given $\alpha \in H^{k}(T, \mathbf{Z})$, there is no natural way to identify a torsion part of $\alpha$ unless $\alpha$ is itself a torsion element of $H^{k}(T, \mathbf{Z})$. On the other hand, if one wishes to study $\alpha$ modulo torsion, this is naturally done by studying the image $\varrho(\alpha)$ of $\alpha$ in $H^{k}(T, R)$.

2.2. Group Cohomology and Classifying Spaces. In order to define the cohomology of a topological group $G$, we first have to introduce the concept of a classifying space. A classifying space $B G$ is the base space of a principal $G$ bundle $E G$, the socalled universal bundle, which has the following fundamental property: Any principal $G$ bundle $E$ over a manifold $M$ allows a bundle map into the universal bundle, and any two such morphisms are smoothly homotopic. We will write

$$
\gamma: M \rightarrow B G
$$

for the induced map of the base manifolds, the so-called classifying map. The topology of the bundle $E$ is completely determined by the homotopy class of the classifying map $\gamma$. That is, the different components of the $\operatorname{space} \operatorname{Map}(M, B G)$ correspond to the different bundles $E$ over $M$. It can be shown that up to homotopy $B G$ is uniquely determined by requiring $E G$ to be contractible. That is, any contractible space with a free action of $G$ is a realization of $E G$. In general the classifying space $B G$ of a compact group is an infinite-dimensional space as the simple examples $B \mathrm{Z}_{2}=R P^{\infty}, B U(1)=C P^{\infty}$, and $B S U(2)=H P^{\infty}$ show. We notice that for our class of Lie groups, $B G$ will be a fibre bundle over $B \Gamma$ with fibre $B G_{0}$.

The group cohomology of a group - as opposed to its cohomology as topological space - can now be defined as the cohomology of the associated classifying space $B G$. Of course, the group cohomology and the ordinary cohomology of $G$ are intimitely related, and one relation between them will be important in Sect. (4). The elements in $H^{*}(B G, \mathbf{Z})$ are also called universal characteristic classes, since under the pullback $\gamma^{*}$ they give rise to cohomology classes in $H^{*}(M, \mathbf{Z})$ that depend only on the pullback $\gamma^{*}$ they give rise to cohomology classes in $H^{*}(M, \mathbf{Z})$ that depend only on the topology of the bundle $E$. 
For a compact Lie group we have the very useful property, due to Borel, that with real coefficients all odd cohomology vanishes:

$$
H^{\text {odd }}(B G, R)=0 \text {. }
$$

So the odd cohomology (and homology) consists completely of torsion. For finite groups an even stronger result holds: all cohomology is finite: $H^{*}(B G, R)=0$. With the use of the exact sequence $0 \rightarrow \mathbf{Z} \rightarrow R \rightarrow R / \mathbf{Z} \rightarrow 0$ this implies for finite $G$ the isomorphism

$$
H^{k}(B G, \mathbf{Z}) \cong H^{k-1}(B G, R / \mathbf{Z})
$$

For the even, real cohomology an important isomorphism exists due to Weil:

$$
H^{*}(B G, R) \cong I(G) \text {. }
$$

Here $I(G)$ is the ring of polynomials on $\operatorname{Lie}(G)$ which are invariant under the adjoint action of $G$. The isomorphism is established using the Chern-Weil homomorphism that maps a polynomial $P \in I(G)$ to the class $[P(F)]$, where $F$ is the curvature of an arbitrary connection in the universal bundle. $P(F)$ is a closed differential form of degree $2 k$ is the polynomial $P$ is of degree $k$. It is a fundamental result that the image $[P(F)]$ in $H^{*}(B G, R)$ is independent of the choice of connection. In this paper we will be mainly interested in the case $k=2$, where $P$ is an invariant quadratic form on $\operatorname{Lie}(G)$, which we usually denote as $\operatorname{Tr}$.

The group cohomology of the unitary groups $U(n)$ is perhaps the most familiar example. It contains no torsion, and is given by the polynomial ring in the Chern classes $c_{k}$ of degree $2 k$,

$$
H^{*}(B U(n), \mathbf{Z})=\operatorname{Pol}\left[c_{1}, \ldots, c_{n}\right] .
$$

As an example of a finite group, we can consider the cyclic group $\mathbf{Z}_{n}$. Again the cohomology ring is finitely generated. There is a single generator $x$ of order $n$ and degree 2, so that

$$
H^{\text {odd }}\left(B \mathbf{Z}_{n}, \mathbf{Z}\right)=0, \quad H^{\text {even }}\left(B \mathbf{Z}_{n}, \mathbf{Z}\right)=\mathbf{Z}_{n} .
$$

Finally, we recall that for discrete groups the cohomology groups $H^{k}(B G, F)$ have an algebraic description, that is perhaps more familiar to the reader. Cochains are represented as functions $\alpha: G^{k} \rightarrow F$, and, if we write the abelian group $F$ multiplicatively, the coboundary operator is defined as

$$
\begin{aligned}
\delta \alpha\left(g_{1}, \ldots, g_{k+1}\right)= & \alpha\left(g_{1}, \ldots, g_{k}\right)^{(-1)^{k+1}} \alpha\left(g_{2}, \ldots, g_{k+1}\right) \\
& \times \prod_{i=1}^{k} \alpha\left(g_{1}, \ldots, g_{i} g_{i+1}, \ldots, g_{k+1}\right)^{(-1)^{i}} .
\end{aligned}
$$

These cochains can be assumed to be normalized, i.e. $\alpha\left(g_{1}, \ldots, g_{k}\right)=1$ if $g_{i}=1$ for some $i$. The equivalence between algebraic cocycles and simplicial cocycles of $B G$ is proved using Milnor's construction of $B G$ [14]. We will give an elementary derivation of this result in Sect. (6) where we treat Chern-Simons theory for finite groups. 


\section{Topological Actions}

As we have already discussed in some detail in the introduction, the form of the standard Chern-Simons action for a trivial bundle $E$ over a three dimensional manifold $M$ with a compact, simple gauge group $G$ is

$$
S(A)=\frac{k}{8 \pi^{2}} \int_{M} \operatorname{Tr}\left(A \wedge d A+\frac{2}{3} A \wedge A \wedge A\right) .
$$

It is a (non-trivial) result in cobordism theory that any closed, oriented 3-manifold $M$ is the boundary of some 4-dimensional oriented manifold $B$, and the trivial bundle $E$ can of course always be extended to a bundle over $B$. This implies that the above expression can be rewritten in terms of the bounding manifold $B$ as

$$
S(A)=\frac{k}{8 \pi^{2}} \int_{B} \operatorname{Tr}(F \wedge F)(\bmod 1),
$$

with $F$ the curvature of any gauge field $A^{\prime}$ on $B$ that reduces to $A$ at the boundary $\partial B=M$. Since the right-hand side is an integer when evaluated on a closed 4-manifold, this form of the action is independent of the choice of bounding manifold $B$ and connection $A^{\prime}$.

If the bundle $E$ is not topologically a product $G \times M$, the above representation needs to be modified. It will in general not be possible to extend the bundle $E$ to a similar bundle over the bounding 4-manifold $B$. To deal with this problem, we can be somewhat more general and allow $B$ to be a smooth singular 4-chain, since a differential form can be integrated over any such chain. Since we are looking for a 4-chain $B$ with a bundle $E^{\prime}$ that restricts to $E$ at the boundary $M$, we are actually trying to find a 4-chain in the classifying space $B G$ that bounds the image $\gamma(M)$ of $M$ under the classifying map $\gamma$. The restriction of the universal bundle to this 4-chain would give us the bundle $E^{\prime}$. The obstruction to the existence of such a 4-chain is exactly measured by the image $\gamma_{*}[M]$ in the cohomology ${ }^{1}$ group $H_{3}(B G, \mathbf{Z})$. We note that if the bundle $E$ has an extension over $B$, the connection can always be extended using a partition of unity. For connected, simply connected Lie groups $H_{3}(B G, \mathbf{Z})$ vanishes, and (3.2) can serve as a general definition of the action (3.1) also for bundles with a non-trivial topology. However, for general compact $G$ we have to take this possible obstruction into proper account.

As we mentioned in the previous section, the third homology group, and in fact all odd homology of $B G$, consists only of torsion. This implies that for each bundle $E$ over $M$ there always exist a positive integer $n$ such that

$$
n \cdot \gamma_{*}[M]=0 \text {. }
$$

\footnotetext{
${ }^{1}$ By permitting $B$ to be a general 4-chain, we reduce the problem to homology and avoid having to consider the bordism theory of $B G$. If we require $B$ to be a smooth 4-manifold, the obstruction to the existence of $B$ with a compatible bundle lies in the bordism group $\Omega_{3}(B G, Z)$ [15]. Bordism groups are as generalizations of homology groups. The homology and bordism groups of $B G$ only differ in their torsion. In fact, if $B G$ has no odd torsion one can prove $\Omega_{3}(B G, \mathbf{Z})=H_{3}(B G, \mathbf{Z})$, see also [16]
} 
Stated otherwise, $E$ can be extended to a bundle $E^{\prime}$ over a 4-chain $B$, whose boundary consists of $n$ copies of $M$, such that the restriction of $E^{\prime}$ on all boundary components is isomorphic to $E$. We shall call such a bundle $E$ of order $n$. Of course, it is always possible to choose the connection such that $A^{\prime}$ also reduces to $A$ at $\partial B$, i.e. exactly, not up to a gauge transformation. So we have no problem in defining the action modulo $1 / n$ as

$$
n \cdot S=\frac{k}{8 \pi^{2}} \int_{B} \operatorname{Tr} F \wedge F(\bmod 1) .
$$

This makes it clear that our task is to resolve an $n$-fold ambiguity consisting of the ability to add a multiple of $1 / n$ to the definition of $S$. We must resolve this ambiguity, for all possible three manifolds and bundles, in a fashion compatible with factorization and unitarity.

So far, the basic object that we have used is the differential form

$$
\Omega(F)=\frac{k}{8 \pi^{2}} \operatorname{Tr} F \wedge F,
$$

which represents an element $\Omega$ of the de Rham coholomogy group $H^{4}(B G, R)$. This differential form has integral periods, so it is in the image of the natural map $\varrho: H^{4}(B G, \mathbf{Z}) \rightarrow H^{4}(B G, R)$. Thus, there exists a cohomology class $\bar{\omega} \in H^{4}(B G, \mathbf{Z})$ such that $\varrho(\bar{\omega})=\Omega$. However, the choice of $\bar{\omega}$ may not be unique. It is unique only modulo a torsion element in $H^{4}(B G, \mathbf{Z})$. We will now show that the choice of a particular $\bar{\omega}$ such that $\varrho(\bar{\omega})=\Omega$ gives a way to resolve the ambiguity in the definition of the action in (3.4). This should not come as a surprise, since the torsion part of $\mathrm{H}^{4}$ is related to the torsion in $\mathrm{H}_{3}$ through the universal coefficient theorem.

Let $\omega$ be any integer-valued cocycle representing the cohomology class $\bar{\omega}$. Then, we define the topological action for a connection on a bundle of order $n$ to be

$$
S=\frac{1}{n}\left\{\int_{B} \Omega(F)-\left\langle\gamma^{*} \omega, B\right\rangle\right\}(\bmod 1),
$$

with $\gamma$ the classifying map $B \rightarrow B G$. (Note that $\left\langle\gamma^{*} \omega, B\right\rangle$ is an integer for all chains $B$.) We can now perform some consistency checks on this definition. First on closed 4-manifolds we have

$$
\int_{B} \Omega(F)=\left\langle\gamma^{*} \omega, B\right\rangle
$$

so that (3.5) is manifestly independent of the bounding manifold $B$ and the way we have continued the bundle and the connection on $B$. It is not difficult to verify that our definition is also invariant under homotopy transformations of the classifying map $\gamma$. Also, the action depends only on the cohomology class $\bar{\omega}$ and not on the particular cocycle chosen to represent it, since under shifts $\omega \rightarrow \omega+\delta \varepsilon$, with $\varepsilon$ an integer cochain, the action changes by

$$
\delta S=-\frac{1}{n}\left\langle\gamma^{*} \delta \varepsilon, B\right\rangle=-\left(\gamma^{*} \varepsilon, M\right\rangle=0(\bmod 1) .
$$

A further necessary requirement will be gauge invariance, at least on manifolds without boundary. Let us first establish that result, before we discuss manifolds 
with boundary in the next section. Let $A$ and $A^{g}$ be respectively a specific connection on $E$ and its gauge transform. Since we can construct an interpolating gauge field $A_{t}$ with $t \in[0,1]=I$, such that $A_{0}=A$ and $A_{1}=A^{g}$, on the manifold $B=M \times I$, we have

$$
S(A)-S\left(A^{g}\right)=\int_{B} \Omega(F) .
$$

The gauge field $A$ is equal up to a gauge transformation at the two ends of the "cylinder" $B$ and we can identify the two ends of $B$ and consider $A$ to be a connection on a bundle $E^{\prime}$ over the closed manifold $M \times S^{1}$. This bundle is constructed with transition function $g$, and $\int_{B} \Omega(F)$ equals the characteristic class of $E^{\prime}$ and is therefore an integer.

We note that the phase choice made in (3.5) is very sensitive to the torsion information in $\omega$. If we transform $\omega \rightarrow \omega+\omega^{\prime}$, where $\omega^{\prime}$ is a $n$-torsion element, the action will pick up a $\mathbf{Z}_{n}$ phase. This is in particular relevant if $\Omega(F)=0$, i.e. "level" $k=0$, as is always the case for finite $G$. Then the class $\omega$ is torsion and determines a 3-cocycle $\alpha \in H^{3}(B G, R / Z)$ through the isomorphism

$$
\operatorname{Tor} H^{4}(B G, \mathbf{Z}) \cong H^{3}(B G, R / \mathbf{Z}) \text {. }
$$

In that case we can rewrite the action, which is now independent of the connection, as

$$
S=\left\langle\gamma^{*} \alpha,[M]\right\rangle
$$

3.1. Differential Characters. The particularly simple formula of (3.10) holds when $\omega$ is a torsion element, but in a certain sense it can be generalized to arbitrary $\omega$. That is, the action $S$ can be rather naturally written as

$$
S=\left\langle\alpha_{A},[M]\right\rangle,
$$

where $\alpha_{A}$ is a 3-cocyle in $H^{3}(M, R / Z)$ that depends on the connection $A$ and the bundle $E$. The cocycle $\alpha_{A}$ is the pull-back under $\gamma^{*}$ of a cochain $\alpha \in C^{3}(B G, R / \mathbf{Z})$ on the classifying space $B G$ which is defined as the modulo 1 reduction of a real cochain $\beta$ that satisfies

$$
\delta \beta=\Omega\left(F_{u}\right)-\omega .
$$

It is evident that $\beta$ and therefore $\alpha$ depend in this way on the torsion information in $\omega$. Here $F_{u}$ is the curvature of a so-called universal connection $A_{u}$ on the classifying space. These universal connections were shown to exist for compact Lie groups by Narasinhan and Ramanan [17], and they have the fundamental property that any connection $A$ on a $G$ bundle $E$ over the manifold $M$ can be obtained as $A=\gamma^{*} A_{u}$ for a suitable classifying map. Although $\alpha$ is evidently not closed and not uniquely determined by the above equation, the pull-back $\gamma^{*} \alpha=\alpha_{A}$ is a well-defined cocycle on $M$ determined completely by the class $\omega \in H^{4}(B G, \mathbf{Z})$ and the connection $A$. In fact, $\alpha$ is what Cheeger and Simons call a "differential character" [4]. Let us explain this in somewhat more detail.

The ring of differential characters $\hat{H}^{*}(T, R / \mathbf{Z})$ is a generalization of the cohomology ring. Just like a cocycle, a differential character $\alpha$ is defined to be a 
homomorphism of the group $Z_{k}(T)$ of singular cycles into $R / \mathbf{Z}$. But instead of requiring it to vanish on boundaries, one imposes the weaker condition that the value assigned to a boundary equals the integral of a certain differential form $\Omega$ (of degree $k+1$ ) on the bounding chain:

$$
\langle\alpha, \partial B\rangle=\int_{B} \Omega(\bmod 1) .
$$

If $\operatorname{dim} T=k$, and $T$ is closed, then the definition of $\hat{H}^{k}$ clearly reduces to the characterization (2.3) of $H^{k}$, because on a $k$-manifold, the $k+1$ form $\Omega$ would automatically be zero. It is furthermore obvious that in order to make the above relation well-defined $\Omega$ should be closed, and that the class $[\Omega]$ in the De Rham cohomology should be necessarily integral. So $[\Omega]$ will always be the image $\varrho(\omega)$ of an integer cocycle $\omega$ under the map $\varrho: H^{k+1}(T, Z) \rightarrow H^{k+1}(T, R)$. Note however that $H^{k+1}(T, \mathbf{Z})$ can contain torsion, and consequently $\omega$ is not completely determined by its image $\varrho(\omega)$.

One can now ask how a differential character can be determined in terms of differential forms and cohomology classes. It is not difficult to proof the following [4]. Let $\Lambda^{k+1}(T)$ denote the set of all pairs $(\Omega, \omega)$ of degree $k+1$ satisfying $[\Omega]=\varrho(\omega)$, then the following sequence is exact:

$$
0 \rightarrow H^{k}(T, R) / \varrho\left(H^{k}(T, \mathbf{Z})\right) \rightarrow \hat{H}^{k}(T, R / \mathbf{Z}) \rightarrow \Lambda^{k+1}(T) \rightarrow 0 .
$$

The proof proceeds by picking a real cochain $\beta$ such that $\beta=\alpha(\bmod 1)$, and defining $\Omega$ and $\omega$ by the relation

$$
\delta \beta=\Omega-\omega .
$$

An important conclusion is that, if $H^{k}(T, R)$ vanishes, the differential form $\alpha$ is completely determined by the pair $(\Omega, \omega)$. This is in particular the case if $T$ equals the classifying space $B G$ and $k=3$, since the cohomology of $B G$ with real coefficient vanishes in three dimensions. The pair $\left(\Omega\left(F_{u}\right), \omega\right) \in \Lambda^{4}(B G)$ determines $\alpha \in \hat{H}^{3}(B G, R / \mathbf{Z})$ uniquely by relation (3.12). When pulled back to the three manifold $\gamma^{*} \alpha$ becomes a cocycle because of dimensional reasons.

3.2. Manifolds with Boundaries. Up to now we only have been able to define the Chern-Simons unambiguously for closed manifolds. However, in analogy with the Wess-Zumino action in group manifold models, the topological action for manifolds $M$ with boundaries can in principle be defined by choosing an arbitrary completion of $M$ to a closed manifold and compute the action of this closed manifold. This prescription depends of course on the choice of completion, but the difference between two choices is completely calculable. This defines the pathintegral on $M$ not as a function but as a section of some (trivial) line bundle over the space of connections on the boundary.

Another approach to manifolds with boundaries is our definition $S=\langle\alpha, \gamma(M)\rangle$ using differential characters. In this light we have to deal with two separate questions: (i) for fixed classifying map $\gamma$, is the topological action well-defined; and (ii) how does it transform under homotopy transformations of $\gamma$ ? In particular, what is the behavior under gauge transformations?

Let us begin to address the first question. We recall that the cochain $\alpha$ in the classifying space $B G$ was defined as the $\bmod \mathbf{Z}$ reduction of a real cochain $\beta$ that 
satisfied $\delta \beta=\Omega\left(F_{u}\right)-\omega$. This relation is not enough to fix $\alpha$ uniquely. In fact, we have the possibility of a "gauge" transformation

$$
\alpha \rightarrow \alpha+\delta v
$$

with $v \in C^{2}(B G, R / \mathbf{Z})$. On closed manifolds this term disappears, but for a 3-manifold $M$ with boundary $\partial M=\Sigma$ the topological action transforms under (3.16) as

$$
S \rightarrow S+\langle v, \gamma(\Sigma)\rangle .
$$

When the boundary of $M$ is non-empty, the path integral on $M$ represents a transition amplitude among initial and final states defined on the boundary of $M$. The addition to the action of the extra term that appears on the right-hand side of (3.17) will make the path-integral on $M$ change by a phase that only depends on the bundle structure and the connection at the boundary $\Sigma$. These phases can be absorbed into the wave functions of the initial and final states. So, although the action is not invariant under (3.16), it transforms in a well-defined way with a boundary term, and the corresponding quantum field theories are identical.

The second question is to what extent is the definition of the action sensitive to homotopy transformations of the classifying map, in particular gauge transformations. First we observe that if we choose for each surface $\Sigma$, with a principal $G$ bundle and a connection, a fixed classifying map $\gamma: \Sigma \rightarrow B G$, and decide to use only these maps at the boundary of our 3-manifold, the action for a manifold with boundary is unambiguously defined. The argument that shows invariance of the action under restricted homotopy transformations of $\gamma: M \rightarrow B G$ that leave $\gamma$ fixed at the boundary is completely analogous to the case of closed manifolds.

However, we still have to deal with gauge invariance. Let us first discuss the case of a trivial bundle $E$ and simple $G$, so that we can use the explicit representation (3.1). It is well-known that the Chern-Simons form

$$
Q(A)=\operatorname{Tr}\left(A \wedge d A+\frac{2}{3} A \wedge A \wedge A\right)
$$

is not invariant under gauge transformations. If $A^{g}$ is the gauge transform of $A$ by a gauge transformation $g$, then

$$
Q\left(A^{g}\right)=Q(A)+d\left(\operatorname{Tr} A \wedge d g g^{-1}\right)-\frac{1}{3} \operatorname{Tr}\left(g^{-1} d g\right)^{3} .
$$

This implies in particular that if $g \neq 1$ at the boundary $\Sigma$, the action transforms under $g$ as

$$
S(A) \rightarrow S(A)+\frac{k}{8 \pi^{2}} \int_{\Sigma} \operatorname{Tr} A \wedge d g g^{-1}-\frac{k}{24 \pi^{2}} \int_{M} \operatorname{Tr}\left(g^{-1} d g\right)^{3},
$$

where the Wess-Zumino term appears. We would like to stress that the variation of $S$ only depends on the connection at the boundary and the gauge transformation $g$. So the path-integral $Z(M)$ transform with the same phase factor as the action $e^{2 \pi i S}$. The result is that $Z(M)$, a function on the space $\mathscr{A}$ of connections at the boundary $\Sigma$, cannot be simply considered to be a function on the space $\mathscr{A} / \mathscr{G}$ of connections modulo gauge transformations, which is the (unconstrained) phase space of the theory. Rather, we should consider it as a section of a line bundle over $\mathscr{A} / \mathscr{G}$. 
For non-trivial topology the argument is analogous. As in the last section, let $\gamma_{0}$ and $\gamma_{1}$ be two homotopic classifying maps that induce the connection $A$ and $A^{g}$ on the manifold $M$. The action will now transform as

$$
S \rightarrow S+\langle\alpha, \gamma(\Sigma \times I)\rangle+\int_{B} \gamma^{*} P(F),
$$

with $B=M \times I$. Here the last term has an interpretation as the Chern-Simons action on $\Sigma \times S^{1}$, where the bundle is constructed by the transition function $g$ restricted to $\Sigma$. This shows that the variation of $S$ only depends on the data on $\Sigma$.

\section{Correspondence with CFT}

Part of the interest of three dimensional Chern-Simons theories comes from their relation [1] to two dimensional current algebra theories. In this section, we will discuss those aspects of this relation that are illuminated by the topological considerations of the last section. In particular, we wish to gain a better understanding of subtleties in this correspondence that arise [2] for groups that are not simply connected. In the subsequent we will assume that the gauge group $G$ is non-abelian.

4.1. The Wess-Zumino Action. To begin with, we recall [5] that conformally invariant sigma models in two dimensions with target space a group manifold require the introduction of the so-called Wess-Zumino term. Let us recall how this is defined. We are given a Riemann surface $\Sigma$ and a map $g: \Sigma \rightarrow G, G$ being some compact Lie group of interest. We wish to define the Wess-Zumino term $S(g)$. To begin with, if $G$ is simply connected, the map $g$ is homotopic to a trivial map, and extends to $g: W \rightarrow G$, where $W$ is a three manifold with $\partial W=\Sigma$. Just as in the formulation (1.3) of the Chern-Simons action, in this situation the Wess-Zumino term has a convenient definition

$$
S(g)=\frac{k}{24 \pi^{2}} \int_{W} \operatorname{Tr}\left(g^{-1} d g \wedge g^{-1} d g \wedge g^{-1} d g\right),
$$

where for reasons explained in [5], $k$ must be an integer. The key object in (4.1) is the differential form $\Phi=\frac{k}{24 \pi^{2}} \operatorname{Tr}\left(g^{-1} d g\right)^{3}$ on the group manifold $G$. This form defines an element of $H^{3}(G, R)$, and since it has integral periods it lies in the image of the natural map $\varrho: H^{3}(G, Z) \rightarrow H^{3}(G, R)$.

If $G$ is not simply connected, the maps $\Sigma \rightarrow G$ come in distinct homotopy classes $\mathscr{U}_{i}$. It may happen, in general, that for suitable $i$, the definition (4.1) does not make sense for $g \in \mathscr{U}_{i}$, since a three manifold $W$ and an extension of $g$ over $W$ may not exist. The obstruction lies in $\mathrm{H}_{2}(G, \mathbf{Z})$. (An example of a semi-simple Lie group with $\mathrm{H}_{2}(G, \mathbf{Z}) \neq 0$ would be $\mathrm{SO}(3) \times S O(3)$, or more generally the groups $\operatorname{Spin}(4 n) / D_{2}$ as discussed in [18].) Even if $W$ does not exist, if we are given two maps $g$ and $g^{\prime}$ both in the same homotopy class $\mathscr{U}_{i}$, the difference $S(g)-S\left(g^{\prime}\right)$ can be defined as in (4.1),

$$
S(g)-S\left(g^{\prime}\right)=\frac{k}{24 \pi^{2}} \int_{W} \operatorname{Tr}\left(\hat{\mathrm{g}}^{-1} d \hat{\mathrm{g}} \wedge \hat{\mathrm{g}}^{-1} d \hat{\mathrm{g}} \wedge \hat{\mathrm{g}}^{-1} d \hat{\mathrm{g}}\right),
$$

where now $W=\Sigma \times I$, and $\hat{g}: W \rightarrow G$ is any map that agrees with $g$ on $\Sigma \times\{0\}$ and with $g^{\prime}$ on $\Sigma \times\{1\}$. Just as in our study of the Chern-Simons term, (4.2) defines the 
Wess-Zumino term except for an integration constant in each topological sector $\mathscr{U}_{i}$. What remains is to fix these integration constants, for all possible $\Sigma$ and all $\mathscr{U}_{i}$, in a way that is compatible with factorization.

If $G$ is semi-simple, $H^{2}(G, R)=0$ and the obstruction to definition (4.1) is the torsion class $g_{*}[\Sigma] \in \mathrm{H}_{2}(G, \mathbf{Z})$. If this class has order $n$, then $S(g)$ can be defined as

$$
S(g)=\frac{1}{n}\left\{\int_{W} \Phi-\left\langle g^{*} \phi, W\right\rangle\right\}(\bmod 1),
$$

with $\partial W=n \cdot \Sigma$ and $\phi$ an integer class in $H^{3}(G, \mathbf{Z})$ such that $\varrho(\phi)=\Phi$. So the torsion information in $H^{3}(G, \mathbf{Z})$ (that gives rise to different "periodic vacua" [18]) suffices to fix the phase ambiguity in the definition of the Wess-Zumino term, completely analogous to our discussion in Sect. (3) of the Chern-Simons action. Thus, for semi-simple $G$, the Wess-Zumino terms - and therefore, according to [5], the conformally invariant sigma models on group manifolds - are classified by $H^{3}(G, \mathbf{Z})$.

In general, when $G$ is not semi-simple the relevant notion is that of differential characters. If $\alpha \in \hat{H}^{2}(G, R / Z)$ is any differential character of $G$ such that $(\Phi, \phi)$ is the corresponding pair in $\Lambda^{3}(G)$, then a general definition of the Wess-Zumino term would be

$$
S(g)=\left\langle g^{*} \alpha,[\Sigma]\right\rangle .
$$

Note that the pair $(\Phi, \phi)$ does not determine $\alpha$ uniquely, and the ambiguities in $\alpha$ correspond to generalized $\theta$ angles on the torus $H^{2}(G, R) / \varrho\left(H^{2}(G, \mathbf{Z})\right)$, as can be seen from Eq. (3.14).

4.2. The Natural Map $H^{4}(B G) \rightarrow H^{3}(G)$. We know now that in general, ChernSimons theories in three dimensions are classified by $H^{4}(B G, \mathbf{Z})$, and WessZumino terms in two dimensions (and hence conformally invariant sigma models) are classified by $H^{3}(G, \mathbf{Z})$. A correspondence between them must therefore involve a natural map from $H^{4}(B G, \mathbf{Z})$ to $H^{3}(G, \mathbf{Z})$. Let us first discuss in geometrical terms the map that proves to be relevant. The universal bundle

$$
G \rightarrow E G \rightarrow B G
$$

gives rise to a map $\tau: H^{k}(B G, F) \rightarrow H^{k-1}(G, F)$, with $F$ any group of coefficients, as follows [3]. Since $E G$ is a contractible space, any cocycle representing an element $\omega \in H^{k}(B G, F)$ becomes exact when lifted to $E G$. So we have a relation of the form

$$
\pi^{*} \omega=\delta \beta \text {. }
$$

We now define $\tau(\omega)$ as the restriction of $\beta$ to the fibre $G$. Since the restriction of $\pi^{*} \omega$ vanishes, the cochain $\tau(\omega)$ is closed and it is easily verified that the cohomology class of $\tau(\omega)$ does not depend on the choice made in the above definition. The inverse of the map $\tau$ is a well-known tool in the study of characteristic classes and cohomology of Lie groups and is usually referred to as transgression [8] (while $\tau$ is also known as the suspension map.)

We now want to show that the map $\tau$ is actually the correspondence between Chern-Simons actions and Wess-Zumino terms that arises in connecting three dimensional quantum field theory with two dimensional quantum field theory. As has been shown in the concluding section of [1], the chiral algebras of two 
dimensional current algebra can be obtained from three dimensions by quantizing the three dimensional Chern-Simons theory on the three manifold $M=D \times R$, with $D$ a disk.

In fact, $[2,20]$, the two dimensional WZW action can be explicitly derived from the three dimensional Chern-Simons action by first integrating over the "time" component $A_{0}$ of the gauge field in the functional integral. The portion of the action (1.1) that depends on $A_{0}$ is

$$
S_{0}=\frac{k}{4 \pi^{2}} \int_{M} \operatorname{Tr}\left(A_{0} \cdot F_{12}\right),
$$

where $F_{12}$ is the spatial component of the curvature, tangent to $D$. The functional integral over $A_{0}$ therefore gives a delta function setting $F_{12}$ to zero, and so we are left with a connection on $M$ whose components tangent to $D$ are pure gauge, i.e. $A_{i}=g^{-1} \partial_{i} g(i=1,2)$ for a map $g: M \rightarrow G(g$ is unique up to a transformation $g \rightarrow u g$ where $u$ depends only on "time"). Since $M$ is contractible, any bundle $E$ over $M$ is necessarily trivial, and we can evaluate the topological action by choosing a global section and pulling the Chern-Simons form to $M$. The resulting action now reads [2]

$$
S=\int_{M} Q\left(g^{-1} d g\right)+\text { exact } .
$$

The exact terms that we will ignore here just correspond to local terms in the two dimensional action. The important contribution is the first term which corresponds to the Wess-Zumino term. With $\theta$ the Maurer-Cartan form on $G$, i.e. the restriction of the connection to the fibre, we can rewrite the first term as

$$
\int_{M} g^{*} Q(\theta)
$$

where $Q(\theta)$ is a closed differential form on $G$ whose class is integer. That is, the Chern-Simons form defines an integer cohomology class in $H^{3}(G, R)$.

The transformation just found from an element of $H^{4}(B G, R)$ with integral periods used to define (1.1) to the element of $H^{3}(G, R)$ with integral periods that appears in (4.7) is precisely the map $\tau$ written out in terms of differential forms. One should go on to show that even when one takes torsion into account, the map from three dimensional theories classified by $H^{4}(B G, \mathbf{Z})$ to two dimensional theories classified by $H^{3}(G, \mathbf{Z})$ is the inverse transgression map. However, we will not tackle this here.

Now, the crucial map $\tau$ from $H^{4}(B G, \mathbf{Z})$ to $H^{3}(G, \mathbf{Z})$ is not necessarily onto. (The special classes in $H^{*}(G)$ that are images of the map $\tau$ are usually referred to as universally transgressive [8].) This fact implies in particular that not all group manifold models "descend" from a three dimensional Chern-Simons theory. In fact, we will see that only those group manifold models that allow a description in terms of a so-called extended chiral algebra will be generated by three dimensional gauge theories.

4.3. Non-Simply Connected Groups. As an example we first consider the cases $G=S U(2)$ and $G=S O(3)$, with the relation

$$
1 \rightarrow \mathbf{Z}_{2} \rightarrow S U(2) \stackrel{\pi}{\longrightarrow} S O(3) \rightarrow 1
$$


For both groups we have $H^{4}(B G)=H^{3}(G)=\mathbf{Z}$. (Here and in the subsequent all cohomology groups are understood to be with integer coefficients unless otherwise stated.) The generators for $H^{4}(B G)$ are respectively the second Chern class $c_{2}$ for $S U(2)$, and the first Pontryagin class $p_{1}$ for $S O(3)$. We will denote the respective generators of the cohomology groups $H^{3}(G)$ by $\alpha$ for $S U(2)$ and $\beta$ for $S O(3)$, with the important relation

$$
\pi^{*}(\beta)=2 \alpha .
$$

This factor of two is simply due to the fact that the volume of $S O(3)$ is half the volume of $S U(2)$. This corresponds to the familiar fact that, if we normalize the Wess-Zumino term with respect to the group $S U(2)$, the corresponding term for $S O(3)$ can only exist for even $k$ [19].

It is well-known that all classes of $H^{*}(S U(2))$ are transgressive [8], so $\tau\left(c_{2}\right)=\alpha$ and this implies a one-to-one correspondence between the $S U(2)$ WZW models and Chern-Simons theories, which are both characterized by their level $k \in \mathbf{Z}$. This will however not be the case for $S O(3)$. In fact, we will see that only the models based on even elements of $H^{3}(S O(3))$, i.e. level $k$ divisible by four, correspond to three dimensional topological theories. Note that it has been observed [2] that exactly for these values the chiral algebra for $S O(3)$ exists, since the chiral vertex operators that are associated with the non-trivial loops in $S O(3)$ have conformal dimensions $k / 4$, and these dimensions should be integer.

This restriction to $k=0(\bmod 4)$ has a completely topological explanation. Let us recall that, although every $S U(2)$ bundle naturally gives rise to a $S O(3)$ bundle, the opposite is not true. Not every $S O(3)$ bundle can be extended to a $S U(2)$ bundle. In fact, this can only happen for certain specific values of the characteristic classes. To determine these values we have to compare the "instanton charges" in the four dimensional $S U(2)$ and $S O(3)$ gauge theories. In a normalization where $S U(2)$ instantons have integer charge the $S O(3)$ instantons can have fractional charges. The fact that a non-simply connected group can have fractional instantons is a well-known phenomenon, e.g. on the hypertorus $T^{4}$ one can construct $S U(n) / \mathbf{Z}_{n}$ instantons with charge $1 / n$ [22]. We will actually show that the minimal charge of a $S O$ (3) instanton is $\frac{1}{4}$, and this naturally quantizes $k$ in units of four. Equivalently, if a $S O(3)$ bundle $E$ on a four manifold extends to a $S U(2)$ bundle, the first Pontryagin class $p_{1}(E)$ always has to be divisible by four. That is, under the map $B \pi: B S U(2)$ $\rightarrow B S O$ (3) as induced by the exact sequence (4.9), we have (see e.g. [21])

$$
B \pi^{*}\left(p_{1}\right)=4 c_{2} .
$$

This can be seen as follows, though perhaps in a slightly abstract way ${ }^{2}$. After lifting to a suitable flag space, any $S U(2)$ vector bundle $V$ (that is, any rank two complex vector bundle of structure group $S U(2))$ splits as a sum of line bundles $V=L \oplus L^{-1}$. Now recall that $p_{1}(E)$ can also be defined as the second Chern class $c_{2}(W)$ of the complexified three dimensional vector bundle $W$ in the adjoint representation of $S O(3)$. In this case we find $W=L^{2} \oplus L^{0} \oplus L^{-2}$, so that $p_{1}(E)=c_{2}(W)=4 c_{2}(V)$.

A concrete example of a $S O(3)$ bundle that has instanton charge $\frac{1}{4}$ can be constructed on $C P^{2}$. Our normalization will be as follows. Let $\lambda_{a}$ denote the

\footnotetext{
${ }^{2}$ We would like to thank D. Freed for the following argument
} 
generators of the Lie algebra of $S O(3)$, satisfying $\left[\lambda_{a}, \lambda_{b}\right]=i \varepsilon_{a b c} \lambda_{c}$. A general curvature can be expressed as $F=\sum_{a} F^{a} \lambda_{a}$ and the instanton number reads

$$
q=\frac{1}{16 \pi^{2}} \int \sum_{a} F^{a} \wedge F^{a} .
$$

For the basic instanton over the 4 -sphere $q=1$. Now consider the fundamental line bundle $L$ over $C P^{2}$. Its curvature $F^{\prime}$ satisfies

$$
\int_{C P^{2}} F^{\prime} \wedge F^{\prime}=4 \pi^{2}
$$

We can now make $L$ into a $S O(3)$ bundle using the embedding $U(1) \subset S O(3)$, which maps $e^{i \theta} \rightarrow e^{i \theta \lambda_{3}}$. This gives $F=F^{\prime} \lambda_{3}$ and in this case the contribution in (4.12) is $4 \pi^{2}$ for $a=3$, and zero otherwise, so $q=\frac{1}{4}$, as promised.

The existence of $S O(3)$ bundles of instanton number 1/4 means that in $S O(3)$ Chern-Simons gauge theory, the level $k$ must be divisible by four (in units in which an arbitrary integer is allowed for $S U(2))$. This result was first established in [2].

We can now easily establish that the $S O(3)$ Chern-Simons theories lead to group manifold models corresponding to even elements of $H^{3}(S O(3))$. Since we have a commuting diagram

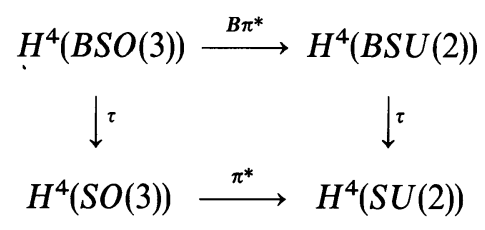

the Eqs. (4.10) and (4.11) immediately imply the relation

$$
\tau\left(p_{1}\right)=2 \beta \text {. }
$$

That $\tau\left(p_{1}\right)$ is necessarily even can also be proved (and generalized to arbitrary $S O(n))$ using the fact that the class $p_{1}$ satisfies $p_{1}=w_{2} \cup w_{2}(\bmod 2)$. This gives $\tau\left(p_{1}\right)=0(\bmod 2)$, since for any coefficent field $F$ the inverse transgression $\tau: H^{k}(B G, F) \rightarrow H^{k-1}(G, F)$ satisfies $\tau(u \cup u)=0$ [3].

Let us now consider the somewhat more general situation where we have an exact sequence

$$
1 \rightarrow Z \rightarrow \widetilde{G} \stackrel{\pi}{\longrightarrow} G \rightarrow 1,
$$

with $\widetilde{G}$ a connected, simply connected, simple group, and $Z$ is a cyclic subgroup of the center of $\widetilde{G}$. In that case all relevant cohomology groups are still isomorphic to Z. The relation between the generators of $H^{3}(\widetilde{G})$ and $H^{3}(G)$ has been carefully investigated in [18]: the constant of proportionality is either one or two. As to the cohomology of the classifying space, let $\tilde{\omega}$ and $\omega$ denote the generators of respectively $H^{4}(B \widetilde{G})$ and $H^{4}(B G)$. In all generality we have a relation

$$
B \pi^{*}(\omega)=N \tilde{\omega},
$$

where we wish to determine the integer $N$. The interpretation will be again that in four dimensions $G$ instantons can have instanton charge $1 / N$ when compared to $\widetilde{G}$ 
instantons, and that the Chern-Simons theory is only well-defined for $k$ divisible by $N$. The calculation of $N$ is as follows. Let $T \subset \widetilde{G}$ be the maximal torus of $\widetilde{G}$ with rank $r$, and let $\Lambda$ be the weight lattice of $\widetilde{G}$ as generated by the fundamental weights $w_{i}$. The inclusion $T \subset \widetilde{G}$ gives a natural map $H^{*}(B \widetilde{G}) \rightarrow H^{*}(B T)$. Now $H^{*}(B T)$ is generated by the 2-cocycles $x_{i}$, the first Chern classes in the decomposition $T=U(1)^{r}$. The $x_{i}$ are the images of the fundamental weights $w_{i}$ under transgression in the universal bundle $E T$, i.e. under the isomorphism $H^{1}(T) \cong H^{2}(B T)$. The image of the generator $\tilde{\omega}$ of $H^{4}(B \tilde{G})$ in $H^{4}(B T)$ is given by the Weyl group invariant combination

$$
\sum_{i, j} \frac{1}{2} A_{i j} x_{i} \cup x_{j}
$$

with $A_{i j}$ the Cartan matrix.

Now let the sublattice $\Lambda^{\prime} C \Lambda$ be the weight lattice of $G=\widetilde{G} / Z$ with generators $v_{i}$. The corresponding elements of $H^{2}(B T)$ we will denote by $y_{i}$. The $y_{i}$ are linear combinations of the $x_{i}$ with integer coefficients. Since $\tilde{\omega}$ is again the smallest Weyl invariant integer combinations of the $y_{i}$, the relation (4.16) between $\tilde{\omega}$ and $\omega$ is simply determined by comparing the images of $\tilde{\omega}$ and $\omega$ in $H^{*}(B T)$. This gives the following result for $N$. Every element $z_{a}$ of the center $Z$ corresponds to a fundamental weight $w_{a}$, and $N$ is defined as the smallest integer that satisfies for all $a$,

$$
\frac{1}{2} N\left\langle w_{a}, w_{a}\right\rangle \in \mathbf{Z} .
$$

This result confirms the relation found in [2] where it was established that the conformal dimensions $h_{a}$ of the vertex operators that create vortices associated to the fundamental group $\pi_{1}(G)=Z$, and that extend the chiral algebra of $\widetilde{G}$ to the chiral algebra of $G$ are given by

$$
h_{a}=\frac{\left\langle k w_{a}, k w_{a}+2 \varrho\right\rangle}{2(k+h)}
$$

with $h$ the dual Coxeter number and $\varrho$ half the sum of positive roots of $\widetilde{G}$. The conformal dimensions $h_{a}$ should be integer and this reproduces the condition $k=0$ $(\bmod N)$ using the relation $2\left\langle\varrho, w_{a}\right\rangle=h\left\langle w_{a}, w_{a}\right\rangle[18]$.

An interesting example is $G=S U(n) / \mathbf{Z}_{n}$. According to [18] the WZW models based on $G$ exist at level $k \in 2 \mathbf{Z}$ or $k \in \mathbf{Z}$ depending on whether $n$ is even or odd respectively. But according to (4.18) the quantization of $k$ for the corresponding Chern-Simons theories is in multiples of $N$, with $N=2 n$ for even $n$ and $N=n$ for odd $n$. So we see that the map $\tau: H^{4}(B G) \rightarrow H^{3}(G)$ is simply multiplication by $n$.

\section{Topological Spin Theories}

Up to now all topological theories were defined on oriented 3-manifolds, possibly with boundary. In general we can consider manifolds with extra structure, and in this section we want to discuss topological theories defined on spin manifolds. We recall that a spin manifold $M$ is an oriented manifold with a choice of spin structure. A spin structure on an oriented manifold exists if the second StiefelWhitney class $w_{2}(T)$ of the tangent bundle $T$ of $M$ vanishes. For three dimensional 
manifolds, this is always so. (But an oriented three dimensional manifold may admit more than one spin structure if there is two-torsion in $H^{1}(M, \mathbf{Z})$.) We will refer to topological theories which require choices of spin structure as "topological spin theories" or simply "spin theories" for short. These theories will have the fundamental property that the definition of partition functions and transition amplitudes associated with $M$ require a choice of spin structure on $M$.

Just as ordinary topological theories in three dimensions lead to ordinary chiral algebras in two dimensions, spin theories lead to what one might call $\mathbf{Z}_{2}$ graded chiral algebras or chiral superalgebras. A chiral superalgebra consists of a collection of holomorphic fields $A_{i}(z)$ of integer or half-integer dimension $h_{i}$ which are closed under operator products,

$$
A_{i}(z) A_{j}(w) \sim \sum_{k} c_{i j}^{k}(z-w)^{h_{k}-h_{i}-h_{j}} A_{k}(w)
$$

(and with $c_{i j}^{k}=0$ unless $h_{k}-h_{i}-h_{j}$ is an integer) and obeying certain other axioms that are just analogous to the axioms for bosonic chiral algebras. If the $A_{i}$ are all of integer dimension, this reduces to the notion of an ordinary (bosonic) chiral algebra. The superconformal algebra in two dimensions should be regarded as a $\mathbf{Z}_{2}$ graded chiral algebra. But there are many other theories that are not superconformal but can be conveniently regarded as theories with $\mathbf{Z}_{2}$ graded chiral algebras.

The general axioms of quantum field theory tell us that a topological spin theory will associate to each two dimensional closed surface $\Sigma$ with a particular spin structure $\alpha$ a Hilbert space $\mathscr{H}_{\Sigma, \alpha^{*}}$. We would like to identify this Hilbert space as the space of holomorphic blocks of a $\mathbf{Z}_{2}$ graded conformal field theory on $\Sigma$. Elementary examples are of course free fermion theories, where the chiral superalgebra is freely generated by the spin $\frac{1}{2}$ currents $\psi_{i}(z)$. These theories possess for a given spin structure only a single holomorphic block whose dependence on the spin structure is given by a theta-function $\vartheta[\alpha](0 \mid \tau)$.

We will not treat here the general theory of "spin" Chern-Simons theories with arbitrary compact gauge group $G$, but restrict ourselves to two examples. Consider first the group $U(1)$, and let $u$ be the generator of $H^{4}(B U(1), \mathbf{Z})$. (Here $u=c_{1}^{2}$, with $c_{1}$ the first Chern class.) Each class $k \cdot u$ defines a topological action, and consequently there are topological $U(1)$ theories in three dimensions with an arbitrary integer level $k$. But if we are given a three manifold with a spin structure, the level need not be an integer; it can be half-integer.

The reason for this is the following. Recall that $H_{3}(B U(1), \mathbf{Z})$ vanishes, so that the action of the $U(1)$ theory on a 3-manifold $M$ can always be defined as

$$
S=\frac{k}{4 \pi^{2}} \int_{B} F \wedge F(\bmod 1)
$$

with $B$ a four manifold that bounds $M$. The curvature form $\frac{1}{2 \pi} F$ represents the first Chern class $c_{1}(L)$ of some complex line bundle $L$ over $M$. This formula for the action is well-defined since the integral

$$
q=\frac{1}{4 \pi^{2}} \int_{B} F \wedge F
$$


is an integer on any closed 4-manifold $B$. But if $B$ is a spin manifold this integer is always even. The reason for this is the following. Equation (5.3) can be interpreted in terms of the intersection pairing in $H^{2}(B, Z)$. In fact, the right-hand side of (5.3) is a de Rham representation of $\left\langle c_{1}(L) \cup c_{1}(L),[B]\right\rangle$. But on a four dimensional spin manifold, the intersection pairing in $H^{2}(B, Z)$ is even, so (5.3) is even. This statement can be given a rather elementary, geometrical proof. Alternatively, one purely analytic way to prove that $c_{1}(L)^{2}$ is even on a four dimensional spin manifold is to note that the index theorem for the Dirac operator $D_{L}$ on a four manifold $B$ twisted by the line bundle $L$ gives

$$
\text { Index } D_{L}=\frac{1}{12} p_{1}(T)+\frac{1}{2} c_{1}(L)^{2} .
$$

( $T$ is the tangent bundle of $B$.) Taking $L$ to be trivial and requiring Index $D_{L}$ to be an integer, we learn that $\frac{1}{12} p_{1}(T)$ is an integer. (In fact, it can be shown to be even.) Generalizing to arbitrary $L$ and requiring that the index should still be an integer, we learn that $\frac{1}{2} c_{1}(L)^{2} \in \mathbf{Z}$, so that $c_{1}(L)^{2}$ and thus (5.3) is even.

Because of this, the definition (5.2) of the action still makes sense modulo 1 for half-integer level $k$ if $M$ is a spin manifold. Note that we tacitly assumed that the spin bordism group $\Omega_{3}^{\text {spin }}(B U(1))$ vanishes, so that both the line bundle and the spin structure of $M$ can always be extended to $B$. This fact is proved by a spectral sequence argument, using the fact that $\Omega_{n}^{\text {spin }}$ (point) $=0$ for $n=1,2,3$ [15], and that $H_{*}(B U(1))$ is torsion free. Thus, we may conclude that there is a topological spin theory with $U(1)$ gauge group and half-integer $k$. These theories should correspond to a $\mathbf{Z}_{2}$ graded chiral algebra in two dimensions. Indeed in our normalization the chiral vertex operators that appear in the two dimensional $U(1)$ chiral algebra have weight $k$. Since we quantize the theory on a Riemann surface with a fixed spin structure, we do not require an implementation of the full modular group, but only of the subgroup which leaves a given spin structure fixed.

Note that $k$ as we define it is half as big as the usual $k$ in most discussions of the abelian theory. Thus, to compare our discussion to other treatments one must make a redefinition $k \rightarrow 2 k$. (However, comparison to [2] needs a redefinition $k \rightarrow 4 k$.) So the "half-integers" become integers, and it is usually said that $k$ must be even in order to define a topological $U(1)$ Chern-Simons theory in three dimensions or in order to be able to define the $U(1)$ chiral algebra in two dimensions (with $\mathbf{Z}_{k}$ fusion rules). Note that in this normalization the $k=1$ theory represents the theory of a free Dirac fermion. In general the bosonic subalgebra of the level $k$ theory equals the chiral algebra at $4 k$, so the spin projection of the Dirac fermion occurs at $k=4$, as is well-known to be true.

A second example of a spin theory is $S O(3)$ Chern-Simons theory. It is likewise true that on a spin 4-manifold the first Pontryagin number of a $S O(3)$ bundle $E$ is always even ${ }^{3}$. Therefore, in the spin category, the level $k$ (normalized with respect to $S U(2))$ can be half as big as in the bosonic category; that is, $k$ can be any even number, not necessarily a multiple of four. So our claim is that for $k=2(\bmod 4)$, the $S O(3)$ affine models do have a chiral superalgebra and have a diagonal partition

\footnotetext{
${ }^{3}$ In fact this is true for all $S O(n)$. It follows from a fact that we used earlier, namely that $p_{1}(E)$ $=w_{2}(E)^{2} \bmod 2$. As a result, the first Pontryagin number of $E$, which is $\left\langle p_{1}(E),[M]\right\rangle$, is equal modulo two to $\left\langle w_{2}(E) \cup w_{2}(E),[M]\right\rangle$, and this vanishes because the intersection form on $H^{2}(M)$ is even for spin manifolds
} 
function if formulated on Riemann surfaces with spin structure. This corresponds to the results in [2]. Indeed, if we calculate the conformal weight $h$ of the chiral field that extends the $S U(2)$ current algebra to $S O(3)$ we find $h=k / 4$, which is halfinteger for $k=2(\bmod 4)$. The extended characters $\tilde{\chi}_{j}$ (with integer spin $j$ ) in the Neven-Schwarz sector are of the form $\tilde{\chi}_{j}=\chi_{j}+\chi_{k / 2-j}$, where the $\chi_{j}$ 's represents the $S U(2)$ characters. If we calculate the partition function of the corresponding bosonic model that is obtained by the summation over spin structures, we find the familiar expressions for the $S O(3)$ partition functions. (See also the discussion in [23].) An elementary example is the case $k=2$ which can be described by three free Majorana fermions. The fermionic model has a single character $(\vartheta[\alpha] / \eta)^{3 / 2}$, and the chiral algebra is generated by the three fermionic currents $\psi_{i}(z)$.

Now let us look at these results in the Hamiltonian formalism. As we have stressed, it must be possible to define the topological action $S$ also on a manifold $M$ with nonzero boundary $\Sigma$, so that one can study transition amplitudes. In that case, the topological action is not defined as a number. Rather, $e^{2 \pi i S}$ must be defined as a section of a line bundle $L$ over the space $\mathscr{A} / \mathscr{G}$ of all gauge-inequivalent connections on $\Sigma$. For illustrative purposes let us consider how these line bundles can be defined in the cases of $S U(2)$ or $S O(3)$ gauge group. For $S U(2)$ the fundamental line bundle over $\mathscr{A} / \mathscr{G}$ is obtained as follows. We take the "twisted" $\bar{\partial}$ operator on $\Sigma$ coupled to two conjugate fields $(b, c)$ of spin 0 and 1 . Here $b$ transforms in the two dimensional representation $R$ of $S U(2)$ and $c$ transforms in the dual representation $\bar{R}-$ which is actually isomorphic to $R$. The line bundle $L$ is now defined as the determinant line bundle of the operator $\bar{\partial}$ coupled to the $S U(2)$ gauge field taken in the two dimensional representation $R$.

Let us now consider $S O(3)$. If we are working on a bosonic surface $\Sigma$, the fundamental line bundle over $\mathscr{A} / \mathscr{G}$ is again the determinant bundle of the $\bar{\partial}$ operator, coupled to a similar pair of fields $(b, c)$ of spin 0 and 1 , which we now put in the adjoint representation of $S O(3)$, which is the lowest dimensional non-trivial representation. The trace over the Casimir of this representation, which determines the anomaly in the two point function of the currents, is four times that of the fundamental representation of $S U(2)$, and this reproduces the result of Sect. (4) that the level $k$ should be a multiple of four. But - and here is the key point - if $\Sigma$ has a spin structure, we can use a single real field $w$ of spin $\frac{1}{2}$, with $w$ in the adjoint representation of $S O(3)$ which is real, rather than the pair $(b, c)$. A single spin $\frac{1}{2}$ field $w$ in the adjoint representation of $S O(3)$ has twice the anomaly of the pair $(b, c)$ in the two dimensional representation of $S U(2)$. So in the spin category $S O(3)$ current algebra can have level $k=2(\bmod 4)$.

\section{Finite Gauge Groups}

We will now turn to the very special case of a finite gauge group $G$. Our main result will be that the structure of topological gauge theories with finite gauge group will correspond to the two dimensional holomorphic orbifold models that were considered in [7]. An holomorphic orbifold is a model obtained by modding out a symmetry group $G$ of a holomorphic or chiral conformal field theory, i.e. a theory whose partition and correlation functions are the modulus squared of a section of a holomorphic line bundle over the moduli space of Riemann surfaces. A famous 
example of such a holomorphic theory is the $E_{8}$ level one WZW model, and holomorphic orbifolds can for instance be obtained by taking the quotient of this model with any finite subgroup $G$ of $E_{8}$. According to [2] the modular geometry of these orbifold CFT's will be reproduced by Chern-Simons theories whose gauge group is the semi-direct product of $E_{8}$ and $G$. However, here the group $E_{8}$ is essentially used to reproduce a trivial theory in two dimensions, at least for closed surfaces. This can be accomplished much more economically by simply omitting the $E_{8}$ gauge theory, and this leads us naturally to consider Chern-Simons theories with finite gauge group $G$. In our opinion these theories are also of some intrinsic interest, since they are very simple examples of topological "quantum field theories." That is, they provide an elementary illustration of the approach to quantum field theory along the lines of category theory - an approach we will now briefly review, before we turn to the explicit construction of the models.

6.1. Axioms of Quantum Field Theory. In a convenient axiomatization of quantum field theory [6], the structure of a $d+1$ dimensional quantum field theory includes (among other things) a functor $\Phi$ from the category of closed $d$-manifolds into the category of Hilbert spaces. We will use the term manifold somewhat loosely; the correct terminology for the objects of our category would be *-manifolds. Here * can be any extra structure: for example an orientation, a spin structure, a complex structure, or a metric. Two manifolds are isomorphic if there exists a diffeomorphism that preserves the structure. Depending on the structure we obtain different types of quantum field theories: topological, "spin," conformal, etc. The "arrows" or morphisms between the objects in these two categories are respectively cobordisms and linear transformations. So an arrow $M: \Sigma \rightarrow \Sigma^{\prime}$ is a $d+1$ dimensional manifold $M$ that interpolates from $\Sigma$ to $\Sigma^{\prime}$. More precisely: $M$ is a manifold that satisfies

$$
\partial M=\Sigma \cup\left(-\Sigma^{\prime}\right),
$$

and whose structure reduces to the respective structures on $\Sigma$ and $-\Sigma^{\prime}$ at its boundary. Here " $\cup$ " denotes the disjoint sum, and $-\Sigma$ the manifold $\Sigma$ with inverse structure, e.g. reversed orientation, as defined by

$$
\partial(\Sigma \times I)=\Sigma \cup(-\Sigma) .
$$

By definition $M$ exists iff $\Sigma$ and $\Sigma^{\prime}$ are cobordant in the category under consideration. Note that we have split the boundary of $M$ into two components $\Sigma$ and $\Sigma^{\prime}$, by labeling them respectively as "ingoing" and "outgoing."

A category also presumes the existence of an associative composition of arrows, which in this case corresponds to "glueing" together two $d+1$ dimensional manifolds $M: \Sigma \rightarrow \Sigma^{\prime}$ and $M^{\prime}: \Sigma^{\prime} \rightarrow \Sigma^{\prime \prime}$ along their common boundary $\Sigma^{\prime}$ to form the manifold $M \circ M^{\prime}: \Sigma \rightarrow \Sigma^{\prime \prime}$. We will further need for each $\Sigma$ an identity arrow $1_{\Sigma}: \Sigma \rightarrow \Sigma$, that satisfies

$$
\mathrm{I}_{\Sigma} \circ M=M \circ 1_{\Sigma^{\prime}}=M,
$$

for which we choose the manifold $1_{\Sigma}=\Sigma \times I$.

The functor $\Phi$ will associate to each $d$-dimensional manifold $\Sigma$ a vector space that we denote as $\mathscr{H}_{\Sigma}$, and to each arrow $M$ a linear map $\Phi_{M}: \mathscr{H}_{\Sigma} \rightarrow \mathscr{H}_{\Sigma^{\prime}}$. If $\Sigma$ and $\Sigma^{\prime}$ are not homomorphic $\Phi_{M}$ represents a tunneling amplitude between different 
space-like topologies. The functor $\Phi$ should preserve the associate composition law which essentially corresponds to the superposition principle of quantum mechanics. Furthermore, the manifold $\Sigma \times I$ is mapped to the identity map $1: \mathscr{H}_{\Sigma} \rightarrow \mathscr{H}_{\Sigma}$.

Since the boundary can consist of disjoint components, we will further require $\Phi$ to satisfy the following natural conditions: (i) if $\Sigma$ consists of the empty set, the corresponding Hilbert space is one dimensional, and (ii) if $\Sigma$ is the disjoint union of several manifolds, the corresponding Hilbert space is the tensor product:

$$
\mathscr{H}_{\Sigma \cup \Sigma^{\prime}}=\mathscr{H}_{\Sigma} \otimes \mathscr{H}_{\Sigma^{\prime}} .
$$

We observe that a closed $d+1$ manifold $M$ can now be seen as an arrow $M: \emptyset \rightarrow \emptyset$, and accordingly is mapped to a morphism $\Phi_{M}: \mathbf{C} \rightarrow \mathbf{C}$. This implies that we can associate to $M$ a number $Z(M)$, the partition function. The manifold $\Sigma \times I$ can also be considered as an arrow $\Sigma \cup(-\Sigma) \rightarrow \emptyset$. This implies a canonical isomorphism

$$
\mathscr{H}_{-\Sigma} \cong \mathscr{H}_{\Sigma}^{*} .
$$

If $M$ and $M^{\prime}$ have boundary components $-\Sigma$ and $\Sigma$, labeled respectively as "outgoing" and "ingoing," we can glue the two manifolds together at $\Sigma$ to form $M$ ". $\Phi$ should also respect this partial composition. So if $v_{i}$ and $v^{i}$ are conjugate bases in $\mathscr{H}_{\Sigma}$ and $\mathscr{H}_{\Sigma}^{*}$, we have

$$
\Phi_{M^{\prime \prime}}=\sum_{i} \Phi_{M}\left(v_{i}\right) \Phi_{M^{\prime}}\left(v^{i}\right) .
$$

This is the famous "glueing" or "sewing" axiom. It allows us in particular to calculate the dimensions of the Hilbert spaces $\mathscr{H}_{\Sigma}$ by glueing the two ends of the cylinder $\Sigma \times I$ together, which gives

$$
\operatorname{dim} \mathscr{H}_{\Sigma}=\operatorname{Tr}_{\mathscr{H}_{\Sigma}} 1=Z\left(\Sigma \times S^{1}\right) .
$$

6.2. Topological Gauge Theories with Finite Gauge Group. After these preliminaries let us now turn to the somewhat esoteric subject of topological gauge theories with finite gauge groups. We will restrict our discussion here for obvious reasons to three dimensions, but the generalization to other dimensions is completely straightforward. In the theories under consideration the only degree of freedom will be the topology of the principal $G$ bundle $E$ over the manifold $M$. For a discrete group all $G$ bundles are of course necessarily flat, and the topology can only be detected in the possible holonomy around homotopically non-trivial closed curves. Accordingly, $G$ bundles are completely determined by homomorphisms of the fundamental group $\pi_{1}(M)$ of the 3-manifold $M$ into the group $G$, up to conjugation. We will denote both this homomorphism and the corresponding homotopy class of the classifying map $M \rightarrow B G$ as $\gamma$. In accordance with the general discussion in Sect. (3) we choose a class $\alpha \in H^{3}(B G, U(1)) \cong H^{4}(B G, \mathbf{Z})$ as topological action. [Note that in this section we will identify $R / \mathbf{E} \cong U(1)$ with the unit circle in $\mathbf{C}$, and write the cohomology groups accordingly multiplicatively, which might confuse the reader.]

The partition function for a closed 3-manifold $M$ will be defined as the sum over all possible $G$ bundles over $M$, weighted with the action $W=e^{2 \pi i S}$,

$$
Z(M)=\frac{1}{|G|} \sum_{\gamma \in \operatorname{Hom}\left(\pi_{1}(M), G\right)} W(\gamma),
$$


with

$$
W(\gamma)=\left\langle\gamma^{*} \alpha,[M]\right\rangle .
$$

In (6.8), $\pi_{1}(M)$ is defined relative to some choice of base point. We notice that the path-integral is reduced to a finite sum. The weights $W(\gamma)$ are manifestly invariant under diffeomorphisms of $M$. Since all bundles over the 3-sphere are trivial, we have in particular

$$
Z\left(S^{3}\right)=\frac{1}{|G|}
$$

Note that although the isomorphism class of $E$ depends on $\gamma$ only up to conjugacy, we sum over all of $\operatorname{Hom}\left(\pi_{1}(M), G\right)$. This prescription is required for the property ${ }^{4}$

$$
Z(M) \cdot Z\left(S^{3}\right)=Z\left(M_{1}\right) \cdot Z\left(M_{2}\right),
$$

where $M$ is the connected sum of the two manifolds $M_{1}, M_{2}$. This relation follows immediately from two facts: (i) the fundamental group of $M$ equals the free product $\pi_{1}\left(M_{1}\right) * \pi_{1}\left(M_{2}\right)$, and (ii) if $\gamma=\left(\gamma_{1}, \gamma_{2}\right) \in \operatorname{Hom}\left(\pi_{1}(M), G\right) \cong[M, B G]$,

$$
\langle\alpha, \gamma(M)\rangle=\left\langle a, \gamma_{1}\left(M_{1}\right)\right\rangle \cdot\left\langle\alpha, \gamma_{2}\left(M_{2}\right)\right\rangle,
$$

since we can construct a 4-manifold $B$ (the "world-sheet" swept out during the factorization process $M \rightarrow M_{1}+M_{2}$ ) that interpolates from $M$ to $M_{1} \cup M_{2}$. Evaluating $\delta \alpha=1$ on the image of this manifold $B$ into $B G$ gives the required property. The normalization of the partition sum (6.8) is such that

$$
Z\left(S^{2} \times S^{1}\right)=1 \text {. }
$$

Here we used that $\pi_{1}\left(S^{2} \times S^{1}\right)=\mathbf{Z}$ and $W(\gamma)=1$, since any bundle over $S^{2} \times S^{1}$ can be continued over the bounding 4-manifold $B^{3} \times S^{1}$, with $B^{3}$ the 3-ball. Stated otherwise, in the light of (6.43), the Hilbert space $\mathscr{H}_{S^{2}}$ turns out to be one dimensional.

Note that if $\alpha$ represents a non-trivial class, it is a priori possible that the partition function vanishes for a particular manifold. For instance, if $G$ equals the cyclic group $Z_{2}$ and $M=R P^{3}$, there are two possible bundles over $M$, since the fundamental group of $M$ has order two. Now recall that the classifying space $B Z_{2}$ can be represented as $R P^{\infty}$. The non-trivial classifying map corresponds to the imbedding $R P^{3} \subset R P^{\infty}$, which generates the third homology group and is dual to $\alpha$. In this case the two contributions cancel and the partition function vanishes

$$
Z\left(R P^{3}\right)=\frac{1}{2}(1+(-1))=0 .
$$

6.3. Hilbert Spaces and Interactions. An interesting class of objects in any (compact) topological field theory are the dimensions of the Hilbert spaces $\mathscr{H}_{\Sigma_{g}}$ obtained by quantizing the theory on a space-time $\Sigma_{g} \times R$ with $\Sigma_{g}$ a Riemann surface of genus $g$. At first sight "quantization" seems quite elementary in this case, since the classical degrees of freedom are discrete and finite. The phase space is simply the moduli space $\mathscr{V}_{g}$ of $G$ bundles over $\Sigma_{g}$,

$$
\mathscr{V}_{g}=\operatorname{Hom}\left(\pi_{1}\left(\Sigma_{g}\right), G\right) / G \text {. }
$$

\footnotetext{
${ }^{4}$ More generally this definition is necessarily to define a functor as discussed in the previous section that respects the glueing axioms
} 
A representation $\gamma$ of the fundamental group of the Riemann surface consists of elements $\left(g_{i}, h_{i}\right)(i=1, \ldots, g)$ satisfying $\prod_{i}\left[g_{i}, h_{i}\right]=1$, and a $G$ bundle is determined by $\gamma$ up to conjugation. Although $\mathscr{V}_{g}$ is a finite set of points, and naively every point contributes one quantum state, in general we only have an inequality

$$
\operatorname{dim} \mathscr{H}_{\Sigma_{g}} \leqq\left|\mathscr{V}_{g}\right| \text {. }
$$

Let us explain why this is true. The dimensions of the Hilbert spaces can be determined in principle - and here also in practice - by calculating the partition functions $Z\left(\Sigma_{g} \times S_{1}\right)$. In the case of a trivial cocycle, $\alpha=1$, the action $W$ will always be one, and the definition of the partition function is just the suitably normalized sum over all representations of the fundamental group of the three manifold. Representations of $\pi_{1}\left(\Sigma_{g} \times S_{1}\right)$ are given by representations $\gamma=\left(g_{i}, h_{i}\right)$ of the fundamental group $\pi_{1}\left(\Sigma_{g}\right)$ of the Riemann surface, together with an element $k$, the holonomy associated to the factor $S^{1}$, in the common stabilizer subgroup $N_{\gamma}$ of the holonomies $g_{i}, h_{i}$. For a fixed $\gamma$ the prefactor in the partition sum is $\left|N_{\gamma}\right| /|G|$. Since $|G| /\left|N_{\gamma}\right|$ equals the order of the orbit of the representation $\gamma$ under conjugation, the partition sum yields exactly $Z\left(\Sigma_{g} \times S^{1}\right)=\left|\mathscr{V}_{g}\right|$, as expected.

However, if $\alpha \neq 1$ there can be $k \in N_{\gamma}$ such that the action $W(\gamma, k)$ is not equal to one $^{5}$. In fact, the action will always be a one-dimensional representation of $N_{\gamma}$,

$$
W\left(\gamma, k_{1}\right) W\left(\gamma, k_{2}\right)=W\left(\gamma, k_{1} k_{2}\right) .
$$

This relation can be proved by constructing a 4-manifold that has as its boundary three copies of $\Sigma_{g} \times S^{1}$, and which allows a representation of its fundamental group that reduces at the boundary to the representations appearing in (6.17). We can take for this 4-manifold $\Sigma_{g} \times Y$, with $Y$ the two sphere with three holes (the trinion or "pair of pants"). With a suitable orientation the monodromies around the three holes are respectively $k_{1}, k_{2}$, and $k_{1} k_{2}$. The summation over all $k \in N_{\gamma}$ in the partition sum will now give zero if the representation (6.17) is non-trivial. So in that case the bundle over $\Sigma_{g}$ described by $\gamma$ does not contribute a quantum state, and the dimension of the Hilbert space is smaller than expected. This effect has been noted in [2] and can be regarded as a global anomaly.

Now for arbitrary genus and general cocycle $\alpha$ the explicit calculation of the dimensions of the Hilbert spaces for arbitrary genus might be a complicated calculation. However, we can make a shortcut. Any Riemann surface can be obtained by sewing together several copies of the thrice-punctured sphere $Y$. Similarly the 3-manifolds $\Sigma_{g} \times S^{1}$ can be obtained by sewing manifolds of the form $Y \times S^{1}$. Now the manifold $Y \times S^{1}$ is not closed. Its boundary consists of three copies of the 2-torus $\Sigma_{1}=S^{1} \times S^{1}$, and consequently the path-integral on $Y \times S^{1}$ will represent a tri-linear map

$$
\Phi_{Y \times S^{1}}: \mathscr{H}_{\Sigma_{1}} \times \mathscr{H}_{\Sigma_{1}} \times \mathscr{H}_{\Sigma_{1}} \rightarrow \mathbf{C} .
$$

\footnotetext{
${ }^{5}$ Probably the simplest example of this phenomenon occurs when one considers the group $G=\mathbf{Z}_{2}$ $\times \mathbf{Z}_{2} \times \mathbf{Z}_{2}$ and the bundle over the 3-torus obtained by twisting with the three generators of $G$ along the three generating cycles of the fundamental group of the torus. It is easy to verify that there is an element in $H^{3}(G, U(1))$ such that $W=-1$
} 

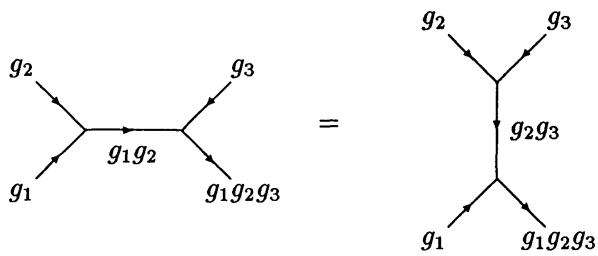

Fig. 1. The sphere with four holes can be composed in two distinct ways from two copies of the sphere with three holes, as indicated in these diagrams. The group elements correspond to monodromies around the punctures

So let us add a few words to the general definition of $\Phi$ in the case of manifolds with boundaries. Let $M$ be an arbitrary 3-manifold $\Sigma \rightarrow \Sigma^{\prime}$. According to our general assumptions $\Phi_{M}$ is a linear map $\mathscr{H}_{\Sigma} \rightarrow \mathscr{H}_{\Sigma^{\prime}}$, and we should specify its matrix elements. For fixed maps $\gamma: \pi_{1}(\Sigma) \rightarrow G$ and $\gamma^{\prime}: \pi_{1}\left(\Sigma^{\prime}\right) \rightarrow G$ the kernel of $\Phi_{M}$ is given by

$$
\Phi_{M}\left(\gamma, \gamma^{\prime}\right)=\frac{1}{|G|} \sum_{\gamma^{\prime \prime} \in \operatorname{Hom}\left(\pi_{1}(M), G\right)} W\left(\gamma^{\prime \prime}\right),
$$

where the summation is over those $\gamma^{\prime \prime}$ that restrict to $\gamma$ and $\gamma^{\prime}$ at the boundaries. It is not difficult to check that this definition satisfies the conditions that we imposed in Sect. (4.1).

So in order to calculate the dimensions of the Hilbert space for arbitrary genus it suffices to know the Hilbert space of the 2-torus and the map $\Phi_{Y \times S^{1}}$ that we just described. Let us first evaluate the action for the manifold $Y \times S^{1}$. Since the manifold has a boundary the action is only well-defined if we choose some fixed classifying maps at the boundaries. So, as discussed in Sect. (3.2), we will assume that for each homomorphism of the fundamental group of a Riemann surface into $G$ we have been given some fixed classifying map. (We will belabor this point in the next section.) An element of $\gamma \in \operatorname{Hom}\left(\pi_{1}\left(Y \times S^{1}\right), G\right)$ is given by elements $g_{i}$ $(i=1,2,3)$ satisfying $g_{1} \cdot g_{2} \cdot g_{3}=1$, that represent the monodromies around the three punctures of $Y$, and an element $h$ that commutes with the $g_{i}$ and that corresponds to the generator of the factor $S^{1}$. So the independent variables are $g_{1}, g_{2}$, and $h$. Of course, the bundle over $Y \times S^{1}$ is only properly defined in terms of the elements $g_{1}, g_{2}, h$ once we picked a base point and specified the cycles along which the holonomies are determined. Let us denote the action as

$$
W(\gamma)=c_{h}\left(g_{1}, g_{2}\right) .
$$

Our claim is that the object $c_{h}$ is an (algebraic) 2-cocycle of the stabilizer group $N_{h} \subset G$, the subgroup of all elements in $G$ that commute with $h$. That is, we have the relation

$$
c_{h}\left(g_{1}, g_{2}\right) c_{h}\left(g_{1}, g_{2}, g_{3}\right)=c_{h}\left(g_{1}, g_{2}, g_{3}\right) c_{h}\left(g_{2}, g_{3}\right)
$$

for any three elements $g_{1}, g_{2}, g_{3} \in N_{h}$. This statement has a very natural geometric proof. Consider the sphere with four holes. It can be obtained in two different ways from two copies of $Y$, as is represented diagrammatically in Fig. 1. This is similarly true after taking the direct product with $S^{1}$. Since the action cannot depend on the 
way we have chosen to construct the manifold, the above relation follows immediately.

So we see that on a priori grounds the Chern-Simons theory associates group cocycles $c_{h}$ to each stabilizer subgroup $N_{h}$ of $G$. However, to actually calculate $c_{h}$ in terms of the 3-cocycle $\alpha$, we have to resort to a different approach.

6.4. A Lattice Gauge Theory Realization. We would now like to explain why the abstract description of the topological action can, in the case of a finite gauge group, be reduced to a concrete description somewhat reminiscent of lattice gauge theory.

Recall that a lattice gauge theory, formulated on a lattice with vertices $V_{i}$, links $L_{i j}$, etc., associates to each link $L_{i j}$, oriented form $V_{j}$ to $V_{i}$, a gauge field $g_{i j} \in G$. A gauge transformation is simply a set of elements $h_{i} \in G$, and the transformation acts on the gauge field as

$$
g_{i j} \rightarrow h_{i} \cdot g_{i j} \cdot h_{j}^{-1} .
$$

The total curvature $f_{i j k}$ for a 2 -simplex is given by the holonomy

$$
f_{i j k}=g_{i j} \cdot g_{j k} \cdot g_{k i},
$$

and is only well-defined modulo conjugation. The action is some local functional of the gauge fields. These lattice theories are particularly well suited for finite groups, where no obvious continuum theory exists. We would like to define here something close to a lattice Chern-Simons theory.

We have seen that for a finite gauge group $G, H^{4}(B G, \mathbf{Z})$ is isomorphic to $H^{3}(B G, U(1))$, and the topological action can be specified by giving an element $\alpha \in H^{3}(B G, U(1))$. Given an oriented three manifold $M$ without boundary (the orientation is always assumed in what follows), and a map $\gamma: M \rightarrow B G$, the topological action is the pairing $\left\langle\gamma^{*} \alpha,[M]\right\rangle$. We will now discuss how this can be evaluated. We may as well assume that $M$ is connected. In addition, we suppose that we are given a triangulation of $M$; given such a triangulation, we will exhibit a recipe for computing the topological action.

Since $B G$ is connected, we can pick a base point $*$ in $B G$, and deform the map $\gamma: M \rightarrow B G$ so that every 0 -simplex in $M$ is mapped to $*$ by $\gamma$. Now let $\sigma$ be a one simplex in $M$. Since the endpoints of $\sigma$ are mapped to the base point $*, \gamma(\sigma)$ is a path from $*$ to $*$ which determines an element of the fundamental group $\pi_{1}(B G)$. On the other hand, this group is isomorphic to $G$ (since $B G$ is the quotient of the contractible space $E G$ by the free action of $G$ ). Thus, to every one simplex $\sigma$ in $M$, the map $\gamma$ determines a group element $g_{\sigma} \in G$. The assignment of group elements to one simplices is reminiscent of lattice gauge theory. In this situation, however, the lattice field strength vanishes: if the three one simplices $\sigma_{1}, \sigma_{2}$, and $\sigma_{3}$ bound a two simplex, then the product $g_{\sigma_{1}} \cdot g_{\sigma_{2}} \cdot g_{\sigma_{3}}$ vanishes (since it represents an element of $\pi_{1}(B G)$, namely $\gamma\left(\sigma_{1} \cup \sigma_{2} \cup \sigma_{3}\right)$, which must vanish since $\sigma_{1} \cup \sigma_{2} \cup \sigma_{3}$ bounds a two simplex or disc). This product is precisely the field strength in the sense of lattice gauge theory. Thus, in this lattice gauge theory model, one is limited to flat connections.

Now, if we really want to establish an analogy with lattice gauge theory, the topological action $\left\langle\gamma^{*} \alpha,[M]\right\rangle$ should depend only the the "gauge field," that is, on 
the $g_{\sigma}$, and not on other details of $\gamma$. In fact, if $\gamma^{\prime}: M \rightarrow B G$ is some other map that determines the same $g_{\sigma}$ 's as those determined by $\gamma$, then $\gamma$ and $\gamma^{\prime}$ are homotopic to each other. To see this, one constructs a homotopy from $\gamma^{\prime}$ to $\gamma$ on the $k$ skeleton of $M$, inductively in $k$. For $k=1$, the existence of a homotopy from $\gamma$ to $\gamma^{\prime}$ is precisely the statement that they determine the same $g_{\sigma}$ 's. Once the homotopy from $\gamma$ to $\gamma^{\prime}$ is established on the $k$ skeleton, the obstruction to extending it over the $k+1$ skeleton lies in $\pi_{k+1}(B G)$ (or more precisely in $H^{k+1}\left(M, \pi_{k+1}(B G)\right)$ ), and vanishes since for $G$ a finite group, the homotopy groups $\pi_{n}(B G)$, for $n>1$, all vanish.

Given that $\gamma$ and $\gamma^{\prime}$ are homotopic, the cocycle condition on $\alpha \in H^{3}(B G, U(1))$ implies that for $M$ a manifold without boundary, the topological action is the same for $\gamma$ as for $\gamma^{\prime}$. Thus, if $M$ has no boundary, the topological action depends only on the "gauge field" $g_{\sigma}$.

6.5. Manifolds with Boundary; Gauge Theory Action. It remains to understand the case in which $M$ has a boundary. If $M$ has a boundary, the topological action cannot be defined as $W=\left\langle\gamma^{*} \alpha,[M]\right\rangle$, because the fundamental class $[M]$ does not exist for a manifold with boundary. Also, we want a somewhat different formulation that will be concrete and closer to lattice gauge theory.

Given a three simplex $T$ and a map $\gamma: T \rightarrow B G$, the cocycle $\alpha \in H^{3}(B G, U(1))$, by definition, assigns an element $W(T) \in U(1)$ to this data. From this point of view, a three simplex $T$ is not just a tetrahedron; it is a tetrahedron with an ordering of the edges as $0,1,2,3$. Roughly, we would like to regard $M$ as a union of three simplices $M=\cup_{i} T_{i}$ and define the topological action as $\prod_{i} W\left(T_{i}\right)$. A chosen triangulation of

$M$ gives a realization of $M$ as a union of tetrahedra. To give an ordering of the vertices in each of these tetrahedra, we order the vertices in $M$ as $1,2,3, \ldots, n$ (if there are $n 0$-simplices in $M$ ), and then in each tetrahedron $T_{i}$, we order the vertices in ascending order.

In a given tetrahedron $T_{i}$, the ordered vertices appear in either a right-handed arrangement or a left-handed arrangement; this determines an orientation of $T_{i}$ which either agrees or disagrees with the orientation induced from that on $M$. Let us define an integer $\varepsilon_{i}$ that is 1 or -1 depending on whether these orientations agree. Then if $M$ has no boundary, the fundamental class of $M$ can be defined as

$$
[M]=\sum_{i} \varepsilon_{i} T_{i} .
$$

It follows from the definition of singular cohomology groups that the topological action, which we earlier defined as $W=\left\langle\gamma^{*} \alpha,[M]\right\rangle$, can equivalently be defined as the product over all individual simplices as

$$
W=\prod_{i} W\left(T_{i}\right)^{\varepsilon_{i}} .
$$

This formula makes sense and is valid whether or not $M$ has a non-empty boundary.

In (6.25) we write the topological action as a product of terms that only depend on the maps to $B G$ of the individual tetrahedra $T_{i}$. This goes in the direction of a lattice gauge theory description, but we have not achieved such a description yet, since in general the $W\left(T_{i}\right)$ do not depend only on the $g_{\sigma}$, as we wish, but on all of the details of the map $\gamma$. 


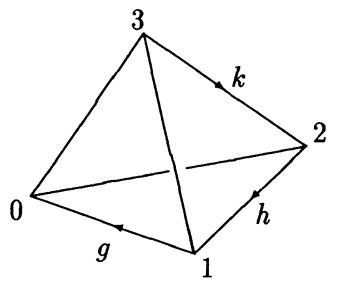

Fig. 2. A 3-simplex $T$ with gauge fields $g, h, k$ has action $W(T)=\alpha(g, h, k)$

To overcome this problem, we proceed as follows. For each choice of the $g_{\sigma}$ 's (with vanishing curvature), we will describe how to pick a particular map $\gamma$ from $M$ to $B G$. By considering only these $\gamma$ 's we will ensure that the $W\left(T_{i}\right)$ 's depend only on the $g_{\sigma}$. The recipe for associating a particular map $\gamma$ with every collection of $g_{\sigma}$ 's is very simple. As in a previous argument, we consider the $k$-skeleton of $M$ and work by induction in $k$. For every homotopy class of paths from $*$ to $*$ in $B G$, that is, for every element $g \in G$, we pick a particular path $u_{g}$, and we agree to use only these paths. This ensures that the map $\gamma$ on the 1-skeleton is uniquely determined by the $g_{\sigma}$ 's. When we consider extending $\gamma$ over the 2 -skeleton, we see that the map to $B G$ of a two simplex $\Delta_{2}$ in $M$ is given by a triple $u_{g_{1}}, u_{g_{2}}$, and $u_{g_{3}}$ (with $g_{1} g_{2} g_{3}=1$ ). For each such triple we pick a particular map $v: \Delta_{2} \rightarrow M$. Similarly, when it comes to the 3 -skeleton, for each three simplex $\Delta_{3}$, the map of its boundary to $B G$ consists of a certain collection of $v$ 's, and for each such collection, we pick a particular map $w: \Delta_{3} \rightarrow B G$. This completes the story for $M$ of dimension three, but otherwise the induction would obviously continue indefinitely.

At this point we have what we want: $(6.25)$ is a formula for the topological action that depends only on the gauge theory data, and is similar to a lattice gauge theory action in that the total action is a product of local terms, one for each threesimplex.

So the basic object is the value of the action $W(T)$ associated to a 3 -simplex $T$. Once we have identified the vertices of the tetrahedron this action is a function of the three independent gauge fields $g, h$, and $k$ on the links, that we can choose as in Fig. 2. We would now like to show that the action

$$
W(T)=\alpha(g, h, k),
$$

is a group cocycle in the algebraic sense, i.e. we would like to prove

$$
\alpha(g, h, k) \alpha(g, h k, l) \alpha(h, k, l)=\alpha(g h, k, l) \alpha(g, h, k l) .
$$

This relation follows quite easily if we consider a 4-simplex with independent gauge fields $g, h, k, l$. Its boundary consists of 5 tetrahedra, and the above equality just expresses the general fact that the action of a boundary vanishes. Note that under a "gauge" transformation $\alpha \rightarrow \alpha \delta \beta$, we have the transformation property

$$
\alpha(g, h, k) \rightarrow \alpha(g, h, k) \frac{\beta(g, h k) \beta(h, k)}{\beta(g, h) \beta(g h, k)} .
$$

Let us summarize our lattice construction. For a given 3-manifold $M$, possibly with boundary, we choose an arbitrary triangulation. [The definition (6.9) of the 
topological action makes it clear that the choice of the triangulation does not matter, though this is not completely obvious in the lattice construction.] We assign gauge fields to the links of the lattice, with the restriction that the curvature vanishes for all 2-simplices that occur in the triangulation. We will sum over all gauge field configurations, modulo gauge transformations that leave one point fixed. This leaves overall conjugation of the gauge fields as a physical degree of freedom. Since flat connections have only non-trivial holonomy around noncontractible loops, our gauge field configurations are labeled by homomorphisms of the fundamental group $\pi_{1}(M)$ into the gauge group $G$. With an arbitrary choice of ordering of the vertices, we associate to three simplex $T_{i}$ the action $W\left(T_{i}\right)$ as in (6.26). The total action is simply the product over all elementary simplices (6.25).

We can now explicitly check some properties of the definition, which are clear on a priori grounds.

First, for a closed manifold, the value of $W$ does not depend on the choice of cocycle used to represent $\alpha \in H^{3}(B G, U(1))$. Under a transformation $\alpha \rightarrow \alpha \delta \beta$ we will pick up terms that are defined on the 2-simplices. These are summed over twice, once in each orientation, and cancel. For example, the simplex depicted in Fig. 2 would transform with (among other terms) a term $\beta(g, h)$ associated to the 2 -simplex labelled by $0,1,2$. However, since the manifold is closed, there will be a neighbouring 3-simplex of opposite orientation that will contribute $\beta(g, h)^{-1}$. So both terms cancel.

It is further not difficult to show that this expression is also invariant under further refinement of the lattice. It is sufficient to consider the barycentric subdivision of a 3-simplex $T$, since every two triangulations have a common subdivision in three dimensions. The barycentric subdivision will replace ' $T$ by 4 new simplices. It is again exactly due to the cocycle condition (6.27) that the sum of the actions of these 4 simplices equals $W(T)$.

Another important property is gauge invariance on closed manifolds. This is due to the fact that a gauge transformation $h_{i} \in G$ on a vertex $V_{i}$ of a simplex $T$ changes the weight $W(T)$ by terms that only depend on the gauge fields on the 2-simplices containing $V_{i}$. If every plaquette belongs to two 3-simplices, as is the case for a closed manifold, the terms cancel two by two. More precisely, a gauge transformation $c \in G$ acting on the vertex $V_{0}$ of the simplex of Fig. 2, will transform the action $W(T)$ as

$$
W(T) \rightarrow \frac{\alpha(c, g, h) \alpha(c, g h, k)}{\alpha(c, g, h k)} W(T) .
$$

Each of the three factors will be cancelled by the neighbouring simplices.

We would like to close this section with one related remark. Depending on the divisability of the order of the group it may be possible to choose the gauge

$$
\alpha\left(g, g^{-1}, h\right)=\alpha\left(g, h, h^{-1}\right)=1 .
$$

In this gauge the above prescription becomes considerably simpler, since now the action $W(T)$ is invariant under chance of labelling of the vertices. One also has the convenient reality condition

$$
\alpha(g, h, k)^{-1}=\alpha\left(k^{-1}, h^{-1}, g^{-1}\right) .
$$




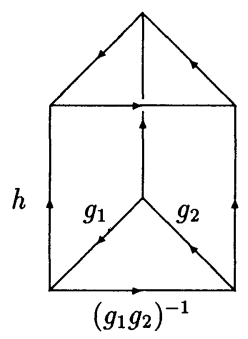

Fig. 3. The manifold $Y \times S^{1}$, the direct product of the 2-sphere with three holes and the circle. The top and bottom and all vertices should be identified. The group elements indicate a homomorphism $\pi_{1}\left(Y \times S^{1}\right) \rightarrow G$
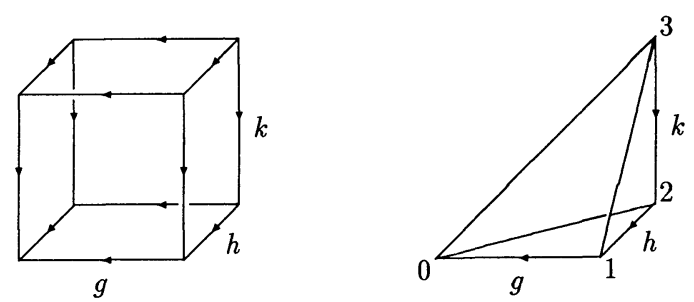

Fig. 4. The 3-torus and one of the six 3-simplices that can be used to triangulate it

6.6. The Partition Function of the 3-Torus. We can now compute several interesting quantities using triangulations. We will first reconsider the manifold $Y \times S^{1}$. We have seen that the Chern-Simons theory associates to each group element $h \in G$ a 2-cocycle $c_{h}$ of the stabilizer group $N_{h}$, and we would now like to express the cocycles $c_{h}$ in terms of the fundamental 3-cocycle $\alpha$. Once we have realized that a sphere with three holes can be represented by a 2-simplex with its three vertices identified, it is not difficult to imagine that $Y \times S^{1}$ can be represented as in Fig. 3. Three simplices suffice to triangulate $Y \times S^{1}$, and the corresponding action is given by

$$
c_{h}\left(g_{1}, g_{2}\right)=\frac{\alpha\left(h, g_{1}, g_{2}\right) \alpha\left(g_{1}, g_{2}, h\right)}{\alpha\left(g_{1}, h, g_{2}\right)} .
$$

It can now be explicitly checked, using repeatedly the cocycle condition $\delta \alpha=1$, that $c_{h}$ is indeed a 2-cocycle of the stabilizer subgroup $N_{h}$. Note that under (6.28) $c_{h}$ transforms as $c_{h} \rightarrow c_{h} \delta \beta_{h}$, with

$$
\beta_{h}(g)=\beta(g, h) \beta(h, g)^{-1} .
$$

Let us now move on to the partition function of the 3-torus $S^{1} \times S^{1} \times S^{1}$. It can be conveniently triangulated with 6 simplices. This can be easily seen when we represent the 3-torus as a cube with periodic boundaries as in Fig. 4 . If $g, h, k$ are the three commuting gauge fields on the edges of the cube, the partition function can be evaluated to give

$$
Z\left(S^{1} \times S^{1} \times S^{1}\right)=\frac{1}{|G|} \sum_{\substack{g, h, k \in G \\[g, h]=[h, k]=[k, g]=1}} W(g, h, k),
$$


with the action given by

$$
W(g, h, k)=\frac{\alpha(g, h, k) \alpha(h, k, g) \alpha(k, g, h)}{\alpha(g, k, h) \alpha(h, g, k) \alpha(k, h, g)} .
$$

We can now explicitly check some general properties of $W$. First we observe that it is indeed invariant under transformations $\alpha \rightarrow \alpha \delta \beta$, in particular $W=1$ whenever $\alpha$ is cohomologically trivial. Furthermore, it can be verified that the above expression is inert under the mapping class group of the 3-torus $S L(3, \mathbf{Z})$.

The action can also be simply rewritten in terms of the 2-cocycles $c_{g}$. Since the three torus can be constructed out of two copies of the manifold $Y \times S^{1}$ we have

$$
W(g, h, k)=c_{g}(h, k) c_{g}(k, h)^{-1} .
$$

This is especially easily visualized with the aid of the triangulated manifolds of Fig. 3 and Fig. 4. We will use this observation to evaluate and interpret the partition function of the 3-torus.

For any finite group $G$ let the positive integer $r(G)$ denote the number of nonisomorphic irreducible representations. It is a familiar fact that $r(G)$ also equals the number of conjugacy classes. Similarly we can define for any 2-cocycle $c$ of $G$ the number $r(G ; c)$ of irreducible projective representations $R(g)$ that satisfy

$$
R(g) R(h)=c(g, h) R(g h) .
$$

It can be shown that $r(G ; c)$ is the rank of the center of the twisted group algebra and that it equals the number of so-called " $c$-regular" conjugacy classes [24], which implies

$$
r(G ; c) \leqq r(G)
$$

An element $g \in G$ is called $c$-regular if $c(g, h)=c(h, g)$ for all $h \in N_{g}$. If $g$ is $c$-regular then so are all its conjugates, and all elements of the form $g^{n} h^{m}$ with $h \in N_{g}$. If we write for fixed $\mathrm{g}$

$$
\varepsilon_{g}(h)=c(g, h) c(h, g)^{-1},
$$

then by a simple computation we can show that $\varepsilon_{g}$ is a one dimensional representation of the stabilizer of $g$. An element $g$ is $c$-regular iff $\varepsilon_{g}=1$. This implies the following expression for $r(G ; c)$ :

$$
r(G ; c)=\frac{1}{|G|} \sum_{\substack{g, h \in G \\[g, h]=1}} c(g, h) c(h, g)^{-1} .
$$

Here we used again the property that the summation $\sum_{k \in K} \varepsilon(k)$ vanishes for any nontrivial one-dimensional representation $\varepsilon(k)$ of a group $K$. Comparing with the expressions (6.34) and (6.36) for the partition function of the 3-torus we obtain

$$
Z\left(S^{1} \times S^{1} \times S^{1}\right)=\sum_{g \in C} r\left(N_{g} ; c_{g}\right)
$$

where $C$ is a set of representatives of the conjugacy classes $C_{A}$ of $G$. So in particular we find that the partition function is an integer, in accordance with its interpretation as the dimension of the Hilbert space associated to the 2-torus $S^{1} \times S^{1}$. 
In this calculation of the Hilbert space for genus one, we recognize the general phenomenon that not all $G$ bundles give rise to quantum states in the theory. According to the result (6.41), only those bundles contribute for which the pair $(g, h)$ satisfies the condition that $h$ is $c_{g}$-regular (or vice versa, the condition is symmetric). So we can take the following basis in the Hilbert space. Let $R_{\alpha}^{g}$ be the irreducible, projective modules of the stabilizer group $N_{g}$ with cocycle $c_{g}$. Since the stabilizer subgroups $N_{g}$ are isomorphic for all $g$ in a conjugacy class $C_{A}$, we can denote these groups as $N_{A}$ and their representations by $R_{\alpha}^{A}$. The basis elements $v_{\alpha}^{A}$ can now be defined by

$$
v_{\alpha}^{A}(g, h)= \begin{cases}\varrho_{\alpha}^{g}(h)=\operatorname{Tr} R_{\alpha}^{g}(h) & \text { if } g \in C_{A}, \\ 0 & \text { otherwise }\end{cases}
$$

Note that the "wave functions" $v_{\alpha}^{A}: \mathscr{V}_{g} \rightarrow \mathbf{C}$ indeed satisfy $v_{\alpha}^{A}(g, h)=0$, if $h \in N_{g}$ is not a $c_{g}$-regular class. It can be verified that this basis is orthonormal. (Recall the manifold $\Sigma_{1} \times I$ furnishes a natural inner product on the Hilbert space $\mathscr{H}_{\Sigma_{1}}$.)

We will now proceed to show that the analysis of this three dimensional topological gauge theory, reproduces the same result obtained in the two dimensional analysis [7] for the dimensions of the Hilbert spaces for arbitrary genus, namely

$$
\operatorname{dim} \mathscr{H}_{\Sigma_{g}}=\sum_{A, \alpha}\left(\frac{\left|N_{A}\right|}{\operatorname{dim} R_{\alpha}^{A}}\right)^{2(g-1)} .
$$

The calculation is not difficult. In [7] the fusion algebra of the two dimensional holomorphic orbifold models was derived, and this was shown to lead to the above dimensions. We only have to check that, when expressed in the basis $v_{\alpha}^{A}$ in $\mathscr{H}_{\Sigma_{1}}$, the morphism $\Phi_{Y \times S^{1}}$ reproduces these fusion rules. This is indeed true, since

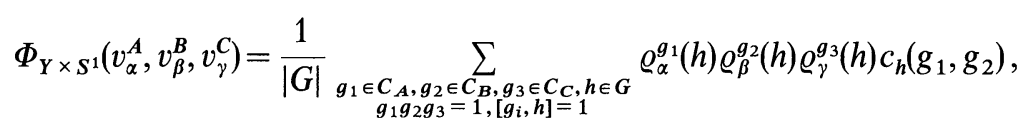

which is completely identical to the fusion algebra that was found in the two dimensional analysis. It is now a straightforward result from the formula of Verlinde [25] that the dimensions of the Hilbert spaces for arbitrary genus are given by Eq. (6.43), see also [7].

A special case occurs when all the cocycles $c_{g}$ are trivial. This is in particular true for abelian $G$, and implies that there exist phases $\varepsilon_{g}(h)$ (defined up to a 1-cocycle) such that

$$
c_{g}(h, k)=\varepsilon_{g}(h) \varepsilon_{g}(k) \varepsilon_{g}(h k)^{-1} .
$$

It is easy to check that the phases $\varepsilon_{g}(h)$ satisfy

$$
\varepsilon_{g^{-1}}(h)=\varepsilon_{g}\left(h^{-1}\right)=\varepsilon_{g}(h)^{-1} .
$$

Let us now introduce the quantities

$$
\sigma(g \mid h)=\varepsilon_{g}(h) \varepsilon_{h}(g)
$$

These objects are manifestly invariant under the transformation (6.28), and so are determined only by the cohomology class $\alpha \in H^{3}(B G, U(1))$ and are invariants of the theory. They equal the phases that were used to describe the modular 
transformation properties of holomorphic orbifold models in [7]. In fact, the genus one modular transformations $S, T$ read in the basis $v_{\alpha}^{A}$,

$$
\begin{gathered}
S_{\alpha \beta}^{A B}=\frac{1}{|G|} \sum_{\substack{h \in C_{B}, g \in C_{A} \\
[h, g]=1}} \varrho_{\alpha}^{g}\left(h^{-1}\right) \varrho_{\beta}^{h}\left(g^{-1}\right) \sigma(g \mid h), \\
T_{\alpha \beta}^{A B}=\delta_{\alpha \beta} \delta^{A B} \varrho_{\alpha}^{g}(g) \varrho_{\alpha}^{g}(1)^{-1} \sigma(g \mid g)^{-1 / 2} .
\end{gathered}
$$

Using surgery with this representation of the modular group as in [1], partition functions of the three dimensional theory can be obtained. For instance we can use genus one Heegaard splittings. A genus $g$ Heegaard splitting [26] is a decomposition of a 3-manifold $M$ into two "handle-bodies" $M_{1}$ and $M_{2}$ of genus $g$ by cutting $M$ along a Riemann surface $\Sigma_{g}$. Such a decomposition is always possible, as an easy triangulation argument shows [26]. The boundaries of $M_{1}$ and $M_{2}$ are identified by a mapping class group element $\Gamma$. Genus one Heegaard splittings with a modular transformation $\Gamma \in S L(2, Z)$ that maps the homology cycle $b$ to $a^{p} b^{q}$ give rise to the Lens spaces $L_{p, q}$. Here $b$ is the generator of the fundamental group of the solid torus. The corresponding partition function is given by

$$
Z\left(L_{p, q}\right)=\left\langle v_{0}, \Gamma v_{0}\right\rangle,
$$

with $v_{0}$ the vacuum state in $\mathscr{H}_{\Sigma_{1}}$. Special Lens spaces are $L_{n, 1}=S^{3} / \mathbf{Z}_{n}$, where we can choose $\Gamma=(T S T)^{n}$. For instance we immediately find [1]

$$
Z\left(S^{3}\right)=S_{00}=\frac{1}{|G|}
$$

in accordance with (6.10). For another concrete example consider again the group $\mathbf{Z}_{2}$. The genus one Hilbert space has 4 states with an $\mathbf{Z}_{2} \times \mathbf{Z}_{2}$ fusion algebra. Since $H^{4}\left(B \mathbf{Z}_{2}, \mathbf{Z}\right)=\mathbf{Z}_{2}$, there are two possible choices for the phase $\sigma(g \mid h)$ : with $g$ the generator of $\mathbf{Z}_{2}$ we have $\sigma(g \mid g)= \pm 1$. We can now calculate the partition function of $S^{3} / \mathbf{Z}_{n}$ using the above representations of $S$ and $T$, with the result

$$
Z\left(S^{3} / \mathbf{Z}_{n}\right)=\left\langle v_{0},(T S T)^{n} v_{0}\right\rangle= \begin{cases}\frac{1}{2}\left(1+( \pm 1)^{n / 2}\right) & n \text { even, } \\ \frac{1}{2} & n \text { odd },\end{cases}
$$

confirming in particular (6.14).

The requirement that the above matrices $S, T$ lead to a consistent representation of the mapping class group leads to certain conditions on $\sigma(g \mid h)$ that were investigated in [7] and are now seen to be solved by giving a cohomology class $\alpha \in H^{3}(B G, U(1))$. For example we can compare our results with the one obtained for the group $G=S_{3}$, where the group of possible phases $\sigma(g \mid h)$ was calculated to be $\mathbf{Z}_{3} \times \mathbf{Z}_{2}$. This result agrees with the three dimensional calculation, since $H^{3}\left(B S_{3}, U(1)\right)=\mathbf{Z}_{6} \cong \mathbf{Z}_{3} \times \mathbf{Z}_{2}$.

6.7. Discrete Torsion. We would like to close with some remarks on discrete torsion [27]. The reader must have noticed a very close similarity of our discussion to the idea of discrete torsion, as it appears in the theory of two dimensional orbifolds. Recall also that discrete torsion is supposed to be classified by the classes $\beta \in H^{2}(B G, U(1))$. Indeed, it has a very natural interpretation as a two dimensional topological theory with finite group $G$, since it associates to each $G$ bundle $E$ over a Riemann surface $\Sigma$ with classifying map $\gamma$ a phase $W(\gamma)=\left\langle\gamma^{*} \beta,[\Sigma]\right\rangle$. 
We would like to briefly clarify why it is true that it is $H^{2}(B G, U(1))$ that classifies discrete torsion. Consider strings propagating on a manifold $M$. For every string world sheet $\Sigma$ ( $\Sigma$ is a Riemann surface perhaps with boundary) and homotopy class of maps $X: \Sigma \rightarrow M$, one wants to find an $R / \mathbf{Z}$ valued topological action $I(X)$ such that

(i) two such actions $I$ and $I^{\prime}$ are considered equivalent if they differ by a functional that only depends on the restriction of $X$ to the boundary of $\Sigma$;

(ii) if $\Sigma$ is a Riemann surface without boundary, and is the boundary of a three manifold $Y$, and if $X$ extends to a map $\hat{X}: Y \rightarrow M$, then $I(X)=0$.

And of course we tacitly assume that the action of a disjoint union of surfaces $\Sigma \cup \Sigma^{\prime}$ is additive, $I\left(\Sigma \cup \Sigma^{\prime}\right)=I(\Sigma)+I\left(\Sigma^{\prime}\right)$. It then follows from (ii) that the topological action is odd under reversal of orientation, $I(\Sigma)=-I(-\Sigma)$, since $\Sigma \cup(-\Sigma)$ is the boundary of the three manifold $\Sigma \times I$.

The requirements (i), (ii) follow from the same consideration as explained in the introduction. The physical reason for requiring (i) is that under the stated condition, the difference between $I$ and $I^{\prime}$ can be absorbed in a redefinition of the wave functions of the initial and final states. As for (ii), it corresponds to factorization. (To see this, bear in mind that the prototype of factorization is a Riemann surface $\Sigma$ splitting into a union of two surfaces $\Sigma_{1}$ and $\Sigma_{2}$. In such a case, a space-time history describing this splitting is a three manifold $X$ such that $\partial X=\Sigma$ $\cup\left(-\Sigma_{1}\right) \cup\left(-\Sigma_{2}\right)$, and the statement of (ii) that $I(\partial X)=0$ amounts then to $I(\Sigma)$ $=I\left(\Sigma_{1}\right)+I\left(\Sigma_{2}\right)$.)

The requirements (i), (ii) above precisely state that $I(X)=\left\langle X^{*} \alpha,[\Sigma]\right\rangle$ for some element $\alpha \in H^{2}(M, \mathbf{Z})$. Therefore, if one is given a sigma model of maps of Riemann surfaces in to a target $M$, and one asks how this model can be modified by weighting the different topological sectors with different phases, the answer is that the possible ways of doing this are classified by $H^{2}(M, Z)$.

Now, suppose that $M$ is obtained as $M_{0} / G$, where $G$ is a finite group which we suppose at first acts freely on $M_{0}$. We suppose that a quantum field theory for maps to $M_{0}$ has already been defined, and we want to define a quantum field theory for maps of Riemann surfaces to $M$. In this process, one has the chance of modifying the naive definition by phases. From what we have just seen, the possible ways of doing this are determined by $H^{2}(M, \mathbf{Z})$. In general, knowledge of $G$ alone does not give complete knowledge of $H^{2}(M, Z)$ (even if one supposes that $G$ acts freely on $M_{0}$ ), and therefore the possibilities for discrete torsion do not reduce simply to a statement about $G$; to give a full statement of the possibilities one needs to consider the nature of $M_{0}$ and compute $H_{2}(M, Z)$.

However, what is usually considered under the heading of discrete torsion are the possibilities that exist universally, just because of taking the quotient by $G$, and irrespective of any properties of $M_{0}$. In this case $M_{0}$ may as well be topologically trivial (contractible). In that case, $M=M_{0} / G$ is a model of $B G$ and $H^{2}(M, \mathbf{Z})$ can be identified with $H^{2}(B G, \mathbf{Z})$. In this way, by considering only the possibilities for discrete torsion that exist universally, only because of the group action, the study of discrete torsion reduces to group cohomology.

Of course, here we have supposed that $G$ acts freely on $M_{0}$ to get a situation that can be conveniently understood geometrically and to make the relevance of group cohomology obvious, without any formulas. It is well known that the formal 
construction of the quantum field theory associated with the quotient of $M_{0} / G$ goes through in much the same way whether $G$ acts freely on $M_{0}$ or not. The formulation and verification of the conditions that arise for modifying the quantum field theory on $M_{0} / G$ with phases likewise makes sense regardless of the nature of the $G$ action, and the possible phases that exist universally are still classified by $H^{2}(B G, \mathbf{Z})$.

As was shown in [7], discrete torsion also gives rise to a natural automorphism of the fusion algebra of a conformal field theory. In the light of the three dimensional framework, this will correspond to a phase transformation of the states in the Hilbert spaces $\mathscr{H}_{\Sigma}$ that is an invariance of all the morphisms $\Phi_{M}$, i.e. all interactions. Let us recall that states $v \in \mathscr{H}_{\Sigma}$ are functions $v(\gamma)$ with $\gamma \in \mathscr{V}$, the moduli space of $G$ bundles on $\Sigma$. An element $\beta \in H^{2}(G, U(1))$ transforms the states as

$$
v(\gamma) \rightarrow v(\gamma)\left\langle\gamma^{*} \beta,[\Sigma]\right\rangle .
$$

However if $\Sigma=\partial M$, as is always the case for a transition amplitude, this phase dissappears because of the condition $\delta \beta=1$.

Acknowledgements. R. D. would like to acknowledge useful discussions with R. Bott, D. Freed, G. Segal, N. Seiberg, C. Vafa, and several colleagues at the Mathematical Institute of the University of Utrecht, and the support of the "Stichting voor Fundamenteel Onderzoek" (F.O.M.) and the Alfred P. Sloan Foundation. The work of E.W. is supported in part by NSF Grant 86-20266 and NSF Waterman Grant 88-17521.

\section{References}

1. Witten, E.: Quantum field theory and the Jones polynomial. Commun. Math. Phys. 121, 351 (1989)

2. Moore, G., Seiberg, N.: Taming the conformal zoo. Phys. Lett. 220B, 422 (1989)

3. Chern, S.-S., Simons, J.: Characteristic forms and geometric invariants. Ann. Math. 99, 48-69 (1974)

4. Cheeger, J., Simons, J.: Differential characters and geometric invariants. In: Geometry and topology. Lecture Notes in Mathematics vol. 1167. Berlin, Heidelberg, New York: Springer 1985

5. Witten, E.: Non-Abelian Bosonization in two dimensions. Commun. Math. Phys. 92, 455 (1984)

6. Segal, G.: Lecture at the IAMP Congress (Swansea, July 1988), and Oxford University preprint (to appear)

7. Dijkgraaf, R., Vafa, C., Verlinde, E., Verlinde, H.: Operator algebra of orbifold models. Commun. Math. Phys. 123, 485 (1989)

8. Borel, A.: Topology of Lie groups and characteristic classes. Bull. A.M.S. 61, 397-432 (1955)

9. Borel, A., Hirzebruch, F.: Characteristic classes and homogeneous spaces I. Am. J. Math. 80, $458-538$ (1958)

10. Milnor, J., Stasheff, J.: Characteristic classes. Annals of Mathematics Studies vol. 76. Princeton, NJ: Princeton University Press 1974

11. Madsen, I., Milgram, R.J.: The classifying spaces for surgery and cobordism of manifolds. Annals of Mathematics Studies vol. 92. Princeton, NJ: Princeton University Press 1979

12. Brown, K.S.: Cohomology of groups. Graduate Texts in Mathematics vol. 87. Berlin, Heidelberg, New York: Springer 1982

13. Freed, D.S.: Determinants, torsion, and strings. Commun. Math. Phys. 107, 483-514 (1986)

14. Milnor, J.: Construction of universal bundles II. Ann. Math. 63, 430-436 (1956)

15. Stong, R.E.: Notes on cobordism theory. Mathematical Notes. Princeton, NJ: Princeton University Press 1968 
16. Conner, P.E., Floyd, E.E.: Differentiable periodic maps. Bull. Am. Math. Soc. 68, 76-86 (1962)

17. Narasinhan, H.S., Ramanan, S.: Existence of universal connections. Am. J. Math. 83, 563-572 (1961); 85, 223-231 (1963)

18. Felder, G., Gawedzki, K., Kupiainen, A.: Spectra of Wess-Zumino-Witten models with arbitrary simple groups. Commun. Math. Phys. 117, 127-158 (1988)

19. Gepner, D., Witten, E.: String theory on group manifolds. Nucl. Phys. B 278, 493-549 (1986)

20. Elitzur, S., Moore, G., Schwimmer, A., Seiberg, N.: Remarks on the canonical quantization of the Chern-Simons-Witten theory. Preprint IASSNS-HEP-89/20

21. Freed, D.S., Uhlenbeck, K.K.: Instantons and four-manifolds. Math. Sci. Res. Inst. Publ. vol. 1, Berlin, Heidelberg, New York: Springer 1984

22. 't Hooft, G.: Some twisted self-dual solutions for the Yang-Mills equations on a hypertorus. Commun. Math. Phys. 81, 167-275 (1981); van Baal, P.: Some results for $S U(N)$ gauge fields on the hypertorus. Commun. Math. Phys. 85, 529 (1982)

23. Schellekens, A.N., Yankielowicz, S.: Extended Chiral algebras and modular invariant partition functions. Preprint CERN-TH5344/89

24. Karpilovsky, G.: Projective representations of finite groups. New York: Marcel Dekker 1985

25. Verlinde, E.: Fusion rules and modular transformations in $2 d$ conformal field theory. Nucl. Phys. B 300, 360 (1988)

26. Hempel, J.: 3-Manifolds. Annals of Mathematics Studies vol. 86. Princeton, NJ: Princeton University Press 1976

27. Vafa, C.: Modular invariance and discrete torsion on orbifolds. Nucl. Phys. B 273, 592 (1986)

Communicated by A. Jaffe

Received June 20, 1989; in revised form October 27, 1989 
